\title{
Instructors' Support of Student Autonomy in an Introductory Physics Course
}

\author{
Nicholas Hall* and David Webb \\ Department of Physics, University of California, Davis, California 95616, USA \\ (Received 31 May 2013; revised manuscript received 9 May 2014; published 26 August 2014)
}

\begin{abstract}
The role of autonomy in the student experience in a large-enrollment undergraduate introductory physics course was studied from a self-determination theory perspective. A correlational study investigated whether certain aspects of the student experience correlated with how autonomy supportive (versus controlling) students perceived their instructors to be. An autonomy-supportive instructor acknowledges students' perspectives and feelings and provides students with information and opportunities for choice while minimizing external pressures (e.g., incentives or deadlines). It was found that the degree to which students perceived their instructors as autonomy supportive was positively correlated with student interest and enjoyment in learning physics $\left(\beta=0.31^{* * *}\right)$ and negatively correlated with student anxiety about taking physics $\left(\beta=-0.23^{* *}\right)$. It was also positively correlated with how autonomous (versus controlled) students' reasons for studying physics became over the duration of the course (i.e., studying physics more because they wanted to versus had to; $\beta=0.24^{* * *}$ ). This change in autonomous reasons for studying physics was in turn positively correlated with student performance in the course $\left(\beta=0.17^{*}\right)$. Additionally, the degree to which students perceived their instructors as autonomy supportive was directly correlated with performance for those students entering the course with relatively autonomous reasons for studying physics $\left(\beta=0.25^{* *}\right)$. In summary, students who perceived their instructors as more autonomy supportive tended to have a more favorable motivational, affective, and performance experience in the course. The findings of the present study are consistent with experimental studies in other contexts that argue for autonomy-supportive instructor behaviors as the cause of a more favorable student experience.
\end{abstract}

DOI: 10.1103/PhysRevSTPER.10.020116

PACS numbers: 01.40.gb, 01.40.Fk

\section{INTRODUCTION}

From the perspective of self-determination theory (SDT) [1-3], to act autonomously or to have autonomy means to experience one's actions as both volitional (i.e., enacted of one's own free will, without outside pressures) and reflectively self-endorsed (e.g., in harmony with one's interests and values). We investigated the degree to which instructors were perceived by their students as supportive of student autonomy in their interactions with students, and with what aspects of the student experience this perceived autonomy support was correlated. We did so to better understand what instructors can do in their interactions with students to improve their experience in the course and what specific aspects of the student experience can be improved as a result of increased autonomy support.

This study was performed within a reformed introductory physics course [4] at the University of California, Davis (UC Davis), a large public university on the West Coast. Some reform efforts in undergraduate physics, based

*nicholasronhall@gmail.com

Published by the American Physical Society under the terms of the Creative Commons Attribution 3.0 License. Further distribution of this work must maintain attribution to the author $(s)$ and the published article's title, journal citation, and DOI. on physics education research (PER), move away from a traditional lecture-based format toward a more interactive format (see, eg., Refs. [5,6]) with more instructor-student interpersonal interactions. Instructors can choose to use this increased interactivity with students either to better support student autonomy (e.g., by framing activities around students' interests and values) or to exert greater control (e.g., by controlling student behavior through directives and commands). In the reformed course of this study, students spend almost 5 hours a week working primarily in small groups in 30-student discussion/labs facilitated by discussion/lab instructors. In this sort of format instructors can provide a range of autonomy support in their interactions with students, making it a good environment in which to investigate autonomy support. This sort of study may not be as fruitful in traditional lecture-based formats in which instructors have fewer opportunities to support student autonomy.

The aspects of the student experience we considered in this study were interest/enjoyment in learning physics, perceived competence in the course, anxiety about taking physics, focus on grades (versus learning), autonomous (versus controlled) reasons for studying physics, and performance in the course. While efforts to enhance student learning as measured by performance are of great value, affective aspects of the student experience may be of particular significance in undergraduate physics and 
astronomy in the United States as the number of physics and astronomy bachelor's degrees earned has remained a small fraction of the bachelor's degrees awarded in the natural sciences. From 2000 to 2009, only $2 \%-3 \%$ of all bachelor's degrees awarded in the natural sciences were in physics and astronomy. In 2009, 3\% were earned in physics and astronomy, compared to $3 \%$ in earth, atmospheric, and ocean sciences, $7 \%$ in chemistry, $9 \%$ in math or statistics, $11 \%$ in agricultural sciences, $21 \%$ in computer sciences, and $47 \%$ in biological sciences (Tables 2-18 of Ref. [7]). Research has argued for the importance of student interest in science, technology, engineering, and mathematics (STEM) as an important predictor of, for example, selecting and persisting in STEM fields (see, e.g., Ref. [8], and references therein).

In this study (study I) we investigated how the autonomy-supportive nature of instructors' interactions with students, as perceived by the students, was correlated with the student experience in the course. In a companion study (hereafter study II, Chap. 3 of Ref. [9]), we investigated how restructuring the entire course, to allow more choices by the students, changed the student experience. Study II was a controlled study in which we compared two course formats, one in which students were given significantly more opportunities for choice than the other.

This paper is organized as follows. In Sec. II, we describe the theoretical background of this study and review relevant literature. In Sec. III, we provide a context for our study, present our hypotheses, and describe the course. In Sec. IV, we describe our research methods and the measures used in this study. In Sec. V, we test each of our hypotheses in turn and present our findings. In Secs. VI and VII, we discuss our main findings and their implications as well as the limitations of this study.

\section{SELF-DETERMINATION THEORY}

In this section we provide the theoretical background of this study by discussing the difference between autonomous and controlled reasons for behavior, positive student outcomes associated with more autonomous reasons for behavior, and ways in which educators can encourage more autonomous reasons for behavior in their students.

\section{A. Autonomous versus controlled reasons for behavior}

We investigated instructors' support of student autonomy from the perspective of self-determination theory [1-3]. SDT is a theory of human motivation that differentiates between autonomous (i.e., self-determined) versus controlled reasons for engaging in an activity. An individual's reasons for engaging in an activity are autonomous when the individual acts volitionally and reflectively endorses their actions.

The prototype of autonomous reasons is intrinsic motivation. When intrinsically motivated, an individual does something because the activity itself is inherently interesting or enjoyable [10]. For example, an intrinsically motivated individual might study a given topic because it is fun or challenging. Humans have a natural tendency to be interested [11], curious [12], and inquisitive and to learn and explore without extraneous incentives [10]. SDT considers the social and environmental factors that either maintain or undermine this tendency to be intrinsically motivated [10].

We all engage in activities that are not inherently interesting or enjoyable to us. SDT distinguishes extrinsic motivation and intrinsic motivation. When extrinsically motivated, an individual does something because it leads to an outcome that is separate from the activity itself [10]. For example, an extrinsically motivated individual might strive to perform well in a course in order to receive praise from others. Extrinsically motivated actions can vary in the degree to which they are autonomous (versus controlled).

SDT differentiates between four types of extrinsic motivation that lie on a relative autonomy continuum $[1,13]$. The least autonomous (most controlling) type of extrinsic motivation is external regulation, whereby an individual engages in an activity to receive a reward (e.g., money for good grades) or avoid punishment (e.g., being grounded for poor grades). Another type of extrinsic motivation is introjected regulation, whereby an individual does something because of internal pressures, such as to avoid guilt or anxiety or to feel pride. Ego involvement $[14,15]$ is a type of introjected regulation in which a person does something for self-esteem reasons such as to feel worthy. For example, a student might study for a test because he would feel bad about himself if he did not study. The internal pressures of introjected regulation are still controlling, though less so than external regulation. Identified regulation is a more autonomous type of extrinsic motivation, whereby an individual identifies with the personal value or importance of a behavior. For example, an individual might engage in an activity as a step toward accomplishing a valued goal. The most autonomous form of extrinsic motivation is integrated regulation, whereby an individual identifies with the personal value or importance of a behavior to the extent that it becomes integrated into the individual's sense of self (i.e., the individual's enduring values and interests). As an example, an individual might study medicine because it will enable her to help those in need around her, congruent with her enduring values and interests [16]. Even though it is autonomous, integrated regulation is not intrinsic motivation because the individual does not engage in an activity because the activity itself is satisfying, but rather because of the activity's instrumental value with respect to an outcome separate from the activity itself [10].

In practice, researchers typically combine external and introjected regulation into controlled reasons for a behavior (i.e., controlled motivation), and intrinsic motivation, 
integrated regulation, and identified regulation into autonomous reasons (i.e., autonomous motivation) [13].

\section{B. More autonomous reasons are associated with a better student experience}

Many studies in educational settings have shown that more autonomous reasons for engaging in an activity are associated with a better student experience and improved academic outcomes from elementary school to medical school. Here, we mention only a few examples. In elementary school more autonomous reasons have been associated with higher performance [17] and higher teacher-rated academic achievement and adjustment in the classroom [18]. More autonomous reasons for taking part in physical education classes among British secondary school students were associated with higher teacher-rated effort and persistence [19]. High school students with more autonomous reasons for attending college reported higher vitality, higher life satisfaction, and lower depression [20]. More autonomous reasons for learning organic chemistry among college students predicted higher interest/enjoyment, higher perceived competence, lower anxiety, and lower likelihood of dropping out [21]. More autonomous reasons for learning about doctor-patient relations among medical students predicted higher perceived competence at interviewing, more internalization of the psychosocial values presented in the course, and interviewing behavior more consistent with these values [22].

\section{Supporting more autonomous motivation}

How do educators encourage more autonomous reasons in their students? Within SDT, autonomy support [1] refers to what one person says and does to enhance another person's subjective experience of autonomy [23]. In this study we refer to autonomy support as provided by an instructor as instructor autonomy support. The SDT concept of autonomy support has primarily been investigated in K-12 contexts, where teachers' motivating styles have been shown to tend toward controlling student behavior (e.g., by relying on extrinsic motivators such as rewards) as opposed to supporting student autonomy (e.g., by nurturing students' interests) (see, e.g., Ref. [24], and references therein). It has been suggested that K-12 teachers often tend toward a controlling instructional style as a reaction to pressures from above (e.g., administrators, state standards), pressures from below (e.g., student passivity), and pressures from within (control-oriented dispositions of the teacher) [24]. Three tasks essential to adopting a more autonomy-supportive style have been outlined, namely, becoming less controlling, appreciating the benefits of an autonomy-supportive style, and learning specific autonomy-supportive instructional behaviors [24].

In this section, we review instructor behaviors that have been identified in the literature, primarily in K-12 contexts, as autonomy supportive. We do so in an effort to be clear about what instructors might do in the classroom to support student autonomy and thus improve the student experience. A review of the literature on autonomy support [23] identified five autonomy-supportive behaviors that have been associated with greater perceived autonomy and activity engagement in individuals. Each behavior has been tested in autonomy support intervention studies. Below we discuss each behavior and what it may look like in practice.

(1) "Provide meaningful rationales," operationally defined as "verbal explanations that help the other person understand why self-regulation of the activity would have personal utility" [23]. This is particularly important when students are asked to engage in an activity they do not find inherently interesting [25]. Providing meaningful rationales refers to communicating to students how the requested activity is useful and meaningful to them (e.g., connects to their values and goals), and thus requires that instructors be aware of students values and goals [25]. It has been recommended [24] that when not able to instantly provide a meaningful rational, another option is to frame the requested activity around intrinsic goals (e.g., self-development) as opposed to extrinsic goals (e.g., social image, financial success, and exam performance) [26,27].

(2) "Acknowledge negative feelings," operationally defined as "tension-alleviating acknowledgments that the request one is making of the other is in conflict with his or her personal inclinations and that his or her feelings of conflict are legitimate (yet not necessarily inconsistent with activity engagement)" [23]. This refers to accepting students' complaints as valid responses to imposed demands and structures, and is particularly relevant when requests, rules, and activities are not in line with students' interests and preferences, as occurs regularly in educational contexts [24]. Statements of negative affect may include the following: this is boring, too much, too hard, just busy work, etc. [24]. Researchers have suggested that "When teachers acknowledge, accept, and even welcome expressions of negative affect, they communicate an understanding of the students' perspectives and put themselves in a position to receive students' negative emotionality as constructive information that can help teachers better (a) align, or realign, students' inner motivation with their classroom activity and (b) transform an instructional activity from 'something not worth doing' (in the eyes of the students) into "something worth doing"' [24]. Acknowledging negative feelings can be extended to encouraging students to work at their preferred pace and allowing or even soliciting students' preferences and critical, independent opinions $[24,28]$.

(3) "Use noncontrolling language," operationally defined as "communications that minimize pressure (absence of 'shoulds,' 'musts,' and 'have tos') and convey a sense of choice and flexibility ..." [23]. This refers to communication that is informational and that helps students find their 
own solutions to motivation problems, as opposed to communication that controls student behavior [24]. Communication that is controlling replaces students' autonomous reasons for acting (i.e., self-regulation) with external (i.e., the teacher's) reasons for acting, whereas autonomy-supportive communication reinforces students' autonomous reasons for acting [24]. Controlling communication includes uttering solutions and answers, uttering directives and commands, criticizing, and asking controlling questions (e.g., "can you do it like I showed you?"); autonomy-supportive communications include offering hints, encouragement, and praise and being responsive to student-generated questions [29].

(4) "Offer choices," operationally defined as "provide information about options, encouragement of choicemaking, and encouragement of the initiation of one's own action" [23]. Research suggests that choice is more likely to enhance student autonomy when students are given choices over their actions (e.g., pace, methods, when to switch activities, and effort) as opposed to choices among options (e.g., which problem to solve) [30], and multiple successive choices as opposed to a single choice [31]. Further, self-determination theory and research findings suggest that choices that are personally meaningful [32] and that allow students to pursue their interests $[33,34]$ and engage in activities they identify as personally valuable or important are most likely to enhance the experience of autonomy. Reference [9] includes an experiment investigating the effects of choice on the student experience and provides a more thorough review of the literature regarding how to offer choice in an autonomysupportive way.

(5) "Nurture inner motivational resources," operationally defined as "vitalization of the other's interest, enjoyment, psychological need satisfaction (autonomy, competence, relatedness), or sense of challenge or curiosity during the engagement of a requested activity" [23]. Additional inner motivational resources include students' preferences, values, and goals. Instructors may first become aware of students' inner resources, for example, through formative assessments or by simply asking what students want or need [24]. Instructors can also encourage students to set their own meaningful goals and encourage initiative and independent learning by having students conduct their own research [24]. Researchers have also argued for "cognitive autonomy support" that encourages students to take ownership of ideas by, for example, encouraging students to share their expertise (e.g., solutions) with each other, allowing plenty of time for decision making, and creating opportunities for students to realign tasks to their interests [35]. A controlling instructional style, on the other hand, would rely on outer sources of motivation (i.e., external pressures and influences), such as directives, incentives, threats of punishment or negative consequences, and deadlines [24]. Research has shown convincingly that the external influence of tangible performance-based rewards, in particular, undermines feelings of autonomy [36-38].

It seems reasonable to expect these five instructor behaviors, primarily investigated in K-12 educational contexts, to be important in supporting autonomous motivation at the college level, which would be consistent with the correlational findings of this study. However, given the many contextual and student differences between K-12 and college, this needs to be tested experimentally at the college level (following, e.g., Ref. [39]). Some of the above behaviors may be more or less important to students' autonomous motivation at the college level, and there may be others of greater relevance to college students.

Other possible autonomy-supportive behaviors include displaying patience to allow time for self-paced learning to occur [24] and providing unconditional positive regard [40]. These have not been tested in autonomy-support intervention studies [23]. In Sec. IV B, we identify the aspects of student-perceived autonomy support we probe in this study.

Various studies in educational settings have shown that autonomy support is important for the maintenance of intrinsic motivation, for extrinsic motivation to become more autonomous (versus controlled), and for various other aspects of the student experience. We mention a few examples here (see, e.g., Ref. [41] for a review). Public elementary school teachers who on a self-report measure were oriented more toward autonomy support had students who were more intrinsically motivated and had higher perceived competence and higher self-esteem than students assigned to more control-oriented teachers [42]. Fourth- through sixth-grade children who perceived their environment as more autonomy supportive reported significantly higher self-esteem, perceived competence, and intrinsic motivation and more control over outcomes [43]. For British secondary school children (mean age of 12.0) in physical education classes, higher perceived autonomy support corresponded to better satisfaction of the students' psychological needs for autonomy, competence, and relatedness as reported by the students, which in turn corresponded to more autonomous reasons for taking part in the class [19]. The interest of seventhgrade German public school students (mean age of 12.3) varied substantially from one lesson to another and students experienced more interest in lessons in which they perceived the climate as more autonomy supportive [44]. Not only is autonomy support important to many aspects of the student experience, but instructors can also be trained to better support student autonomy. A metaanalysis of 19 studies found that training intervention programs designed to help people (teachers, parents, counselors, etc.) support the autonomy of others were effective overall with a mean weighted [45] effect size of $0.63[23]$. 


\section{OUR CORRELATIONAL STUDY}

In this section, we situate our study within the context of previous work, present our hypotheses, and describe the course in which our study was carried out.

\section{A. Context}

As demonstrated by the literature referenced in Sec. II C, autonomy support as provided by an instructor has primarily been investigated in primary and secondary school settings. The present study is the first to our knowledge to investigate the self-determination theory concept of autonomy support in a college-level physics course. The strong research support for the benefits of autonomy support in other educational settings highlights the potential value of this area of research to the PER community. Additionally, Dancy and Henderson [46], in applying their framework for articulating instructional practices and conceptions to well-known PER-based curricula, identify student autonomy (which they describe as students having input in instructional decisions) as an instructional practice not widely embraced by the PER community. They argue, further, that the evidence in favor of student autonomy warrants a serious investigation by the PER community into what aspects, if any, of student autonomy might be profitably embraced. The present study (study I), investigating how the degree to which students perceive their instructors as autonomy supportive correlates with the student experience, and study II [9], investigating the effects of providing students with additional choice, bear on that question.

Within the broader science education research community, this study is one of only a few studies to investigate autonomy support in a college-level natural science course, with Ref. [21] (hereafter BD00) being the first. The present study builds on that of BD00; thus, we briefly describe that study. The introductory organic chemistry course of BD00 was organized following the Workshop Chemistry Project [47]. According to BD00, in addition to standard full-class lectures, students were randomly assigned to 6-8 member workshops (i.e., intensive study groups) that met once a week for 2 hours, over a period of 13 weeks. Peer leaders, who were either advanced undergraduate or graduate students, led the workshops. The workshops were designed to give students opportunities to participate, receive social support, and solve problems in groups; they are different from traditional recitations in that they focus on active learning as opposed to simply reviewing course materials. The workshop leaders received some training in studentcentered teaching, active engagement, facilitating group problem solving, and peer support. BD00 found that students who perceived their instructors as more autonomy supportive tended to have increases in interest/enjoyment, perceived competence, and autonomous reasons for studying chemistry, as well as decreases in anxiety during the course. Autonomy support as reported by the students also correlated with student performance (where this effect was moderated by students' initial reasons for studying chemistry).

The present study builds on that of BD00 as follows. We perform an independent check of the findings from the correlation study of BD00, and we do so in a context quite different from that of BD00. Differences between the present study and that of BD00 include that they were performed at different universities in different geographical locations (a small Eastern university and a large public Western university), with different student populations, in distinct fields of study (introductory organic chemistry and introductory physics), in different course formats, at different times, and by different sets of researchers. By comparing and contrasting our results with those of BD00, we provide a strong test of their external validity. The sample size of students in the present study is slightly larger than BD00. Also, students were divided among only five instructors in the present study, allowing us to account for and investigate differences among instructors in the dependent variables of our multiple regression models, which was not possible with the 42 workshop leaders of BD00 [48].

Finally, the present study (study I) sets the stage for study II in which we performed a controlled experiment, with a subset of the students in the present study, to investigate the effects of the amount of student choice designed into the course format [9]. Study I investigates student-perceived autonomy support as provided by an instructor. Study II investigates the effects of how supportive the course design is of student autonomy, while controlling for the findings of study I related to autonomy support as provided by an instructor.

\section{B. Hypotheses}

We tested the following hypotheses, derived from the findings of BD00, using data from surveys administered pre and post instruction as discussed below and after controlling for various predictor variables not mentioned here. By more favorable student adjustment, we mean higher interest/enjoyment and perceived competence in learning physics, lower anxiety about taking introductory physics, and less of a focus on grades (versus learning).

Hypothesis 1: More autonomous reasons for studying physics (i.e., more because they want to versus have to) upon entering the course will predict more favorable student adjustment in the course.

Hypothesis 2: The degree to which students perceive their instructors as autonomy supportive will be positively correlated with student adjustment at the end of the course and how autonomous (versus controlled) students' reasons for studying physics are at the end of the course.

Hypothesis 3: The degree to which students perceive their instructors as autonomy supportive will be positively correlated with performance in the course, and this correlation will be moderated by how autonomous students' reasons for studying physics were upon entering the course. 
Hypothesis 4: More positive changes, over the duration of the quarter, in autonomous reasons for studying physics will be positively correlated with performance in the course.

Hypotheses 1 and 4 are consistent with the aforementioned findings that more autonomous reasons for engaging in an activity are associated with a better student experience and improved academic outcomes. Hypotheses 2 and 3 are consistent with the various studies mentioned above showing that autonomy support is associated with more autonomous reasons, greater student engagement, and various other positive aspects of the student experience.

\section{The course}

This study was carried out in the context of an undergraduate introductory physics course for students in the biological sciences at UC Davis during Winter quarter 2010-2011. This course is known as Collaborative Learning through Active Sense-making in Physics (CLASP). CLASP is a three quarter series with course numbers Physics 7A, 7B, and 7C. This study was carried out in Physics 7A with 315 students divided into two lecture sections and 11 discussion/lab (DL) sections. The single course lecturer gave a weekly 80 -minute lecture to each lecture section. Six DL Instructors, who were graduate teaching assistants, taught the $11 \mathrm{DL}$ sections. Each DL section met twice a week for 140 minutes each time and consisted of approximately 30 students. In a typical CLASP course, students spend the majority of their time in DL working through a series of established activities in groups of five at a shared group chalkboard. Group work is interspersed with student presentations of their work and brief whole class discussions facilitated by the DL instructor. See Ref. [4] for a description of the CLASP program.

Reference [4] includes a short discussion of the teaching professional development program all of our DL instructors pass through. The program starts with an intensive threeday introduction to teaching, which includes discussions of videos of actual DL sections showing some exemplary types of interactions between instructors and student discussion groups (five students) and interactions between instructors and the entire class (six groups of five students each) during whole class discussions. The professional development program continues during each DL instructor's first full quarter of teaching with one-hour weekly seminars (taught in an active learning style). These seminars cover general issues of teaching and learning but also include substantial practical work on improving instructors' own students' small group discussions and whole class discussions, grading using rubrics, etc. Although the same curriculum of activities is provided to the DL instructors and they receive the above-mentioned training in facilitating collaborative and active learning in this course, they do take some latitude in how they spend their class time given their preferred instructional techniques.
In the CLASP course of the present study, the DLs were the major component of the course, in terms of both time and content. The vast majority (if not all) of the required content of the course was covered in the DLs. The lectures served primarily to supplement the DLs (e.g., by preparing students for the DLs by defining technical words or elaborating on concepts covered in DL) and to facilitate the administration of weekly quizzes. We expect this to have been quite different from the introductory organic chemistry course of BD00 in which the workshops likely primarily supplemented the lectures.

\section{METHODS}

In this section, we discuss how study participants were selected as well as the various measures used in this work. 315 students were enrolled in the course at the end of the quarter. We excluded from our study population two students who received a final grade of incomplete and two students who did not take the final exam, leaving 311 students. Partway through the quarter instructor 5 decided to offer their two sections in a way quite different from the standard discussion/lab [49], discouraging the use of instructor 5 in our study. Upon further inspection we found significantly different correlations when comparing instructor 5 to all other instructors for hypotheses 1 and 3 . We thus excluded the students of instructor 5 from this study (except in Fig. 1 where instructor 5 is shown for comparison), leaving 261 students. Additionally, only those students who responded to all items on the questionnaires discussed below were included in this work, leaving the 182 students included in our analysis. These 182 students performed better than the 79 who did not respond to all questionnaire items [two-sided $t(142)=3.6, p<0.001$ ] by an average of 0.26 grade points, $95 \%$ CI [0.12, 0.41]. They also had a higher incoming GPA that, when controlled for (as done in our analysis below), resulted in the difference in performance becoming statistically insignificant $(0.13$ grade points, 95\% CI $[-0.01,0.28])$. Finally, as not all students granted the researchers permission to access their GPAs, our models containing GPA had fewer students.

We now describe the three types of measures used in this study to test hypotheses $1-4$. We refer to them as adjustment, autonomy versus control, and performance measures. Adjustment and autonomy versus control measures were composed into pre (hereafter T1) and post (hereafter T2) questionnaires. The T1 questionnaire was administered to the students in the first week of instruction (one part in the first lecture and another part in the second DL). The T2 questionnaire was administered to the students in the last or 10th week of regular instruction (in the last or 18th DL) before the final exam. Self-reported gender was included in the T2 questionnaire and is represented in this work by the control variable Female, which is 0 for males and 1 for females (see Ref. [9] for gender-related analyses and discussion). As in the previous sentence, we capitalize 
all variables throughout this work to distinguish variables (e.g., Female) from non-variable words (e.g., female). When administering both the T1 and T2 questionnaires, it was communicated to the students that the questionnaires were voluntary, did not affect their grade, and that their results would be kept confidential, specifically that their instructors would not see their responses. We administered the questionnaires.

\section{A. Adjustment measures}

Interest/Enjoyment Measure.-This nine-item measure was adapted by BD00 from Ref. [22] and attempts to measure students' interest/enjoyment in learning and studying introductory physics. Students rated on a five-point scale how true four items were (e.g., "I enjoy learning about introductory physics") and how interested and curious they were on five items (e.g., "How interesting is learning about introductory physics?"). The total score (hereafter Interest/ Enjoyment) is the sum of the nine items. This measure has a single underlying factor (BD00), and Cronbach's alphas for the present study were 0.92 at both $\mathrm{T} 1$ and $\mathrm{T} 2$ (see Sec. VII C for a discussion of alpha values obtained in this study).

Perceived Competence Scale.-This five-item measure was adapted by BD00 from Ref. [22] and assesses the students' degree of perceived competence in learning introductory physics. Students were asked to rate on a five-point Likert-type scale how true each item was (e.g., "I have confidence in my ability to be successful in introductory physics"). The total score (hereafter Perceived Competence) is the sum of the five items. Cronbach's alphas for the present study were 0.84 and 0.86 at $\mathrm{T} 1$ and $\mathrm{T} 2$, respectively.

State-Trait Anxiety Inventory.-This validated inventory [50] attempts to measure the amount of state (versus trait) anxiety students feel about taking introductory physics. We used 14 of the 20 state anxiety items (BD00 used one additional item). Students rated how well each item described them on the scale "not at all," "slightly," "moderately," or "a great deal." The total score (hereafter Anxiety) is the sum of the 14 items [51]. Cronbach's alphas for the present study were 0.92 at both T1 and T2.

Grade Orientation Measure.-This four-item measure was created by BD00 to measure the extent to which students are focused more on grades than on learning. Students rated on a five-point scale how true each item was (e.g., "I care much more about what I learn than the grades I get"). The total score (hereafter Grade Orientation) is the sum of the four items, two of which are reversed scored (including the above example). Cronbach's alphas for the present study were 0.63 and 0.66 at T1 and T2, respectively. For another example of the use of orientations in PER, specifically, learning versus performance orientation, see Ref. [52].

These four adjustment measures are provided in the Supplemental Material [53]. All adjustment measures were administered at both T1 and T2. All adjustment measure items of this study were identical to those of BD00 except that references to "organic chemistry" were replaced by "introductory physics." The principal component factor analysis of BD00 found that the 18 items of the Interest/ Enjoyment Measure, Perceived Competence Scale, and Grade Orientation Measure had the expected three-factor solution. Throughout this study, by more favorable adjustment we mean higher Interest/Enjoyment and Perceived Competence and lower Anxiety and Grade Orientation measured as described above.

\section{B. Measures of autonomy versus control}

General Causality Orientations Scale (GCOS).Created by Ref. [54], this validated 36-item questionnaire consists of 12 achievement or socially oriented hypothetical situations (e.g., "You have been offered a new position in a company where you have worked for some time. The first question that is likely to come to mind is:"). There are three ways of responding to each situation: autonomous (e.g., "I wonder if the new work will be interesting?"), controlled (e.g., "Will I make more at this position?"), and impersonal (e.g., "What if I can't live up to the new responsibility?"). As described by Ref. [54], autonomy-oriented individuals seek out opportunities for self-determination and choice, control-oriented individuals seek out or interpret events as controlling, and impersonal-oriented individuals believe outcomes to be independent of their behavior (i.e., they experience their behavior as regulated by impersonal forces, such as luck or fate, rather than personal intentions). Students rated on a seven-point Likert-type scale how likely they would be to respond in each of the three ways. Three subscale scores were calculated by adding the 12 responses for each. The GCOS was administered only at T1 to measure the three general causality (i.e., motivational) orientations of the students before they were influenced by the course. These general motivational orientations were then controlled for to isolate the course-specific orientations of interest in this study. Cronbach's alphas for the present study were $0.74,0.66$, and 0.72 for the autonomous, controlled, and impersonal subscales, respectively.

Learning Self-Regulation Questionnaire (LSRQ).-This 12-item questionnaire was adapted by BD00 from the original Self-Regulation Questionnaire for elementary students [13] and the subsequent version adapted for medical students [22]. The items used here are identical to those used in BD00, with "organic chemistry" replaced by "introductory physics." This questionnaire addresses why students are studying introductory physics on two subscales: autonomous or controlled. Students rated on a fivepoint Likert-type scale how true each reason was for them. Four of the 12 items were either intrinsic or identified and thus considered autonomous (e.g., "The reason that I will work to expand my knowledge of physics is because it's interesting to learn more about the nature of physics"). The remaining eight items were either external or introjected 
and thus considered controlled (e.g., "The reason that I will work to expand my knowledge of physics is because a good grade in physics will look positive on my record"). The total score for each subscale is the sum of the items on that subscale. The LSRQ was administered at T1 and T2. Cronbach's alphas were 0.69 and 0.71 for the autonomous subscale at T1 and T2, respectively, and 0.60 and 0.71 for the controlled subscale at T1 and T2. The LSRQ has been shown to have the expected two-factor solution (BD00). As is common with this measure, we calculated the Relative Autonomy Index (RAI) by subtracting the $z$ score of the controlled subscale from the $z$ score of the autonomous subscale. These $z$ scores were calculated once for all 182 students included in our study. A more positive RAI represents relatively more autonomous (versus controlled) reasons for studying physics. Autonomous reasons correlated with the general autonomous orientation of the GCOS $[r(180)=0.44,95 \%$ CI $[0.06,0.81]]$ and controlled reasons correlated with both the general controlled $[r(180)=0.49,[0.12,0.85]]$ and impersonal $[r(180)=$ 0.62, [0.31, 0.94]] orientations of the GCOS (as in BD00), thus providing additional construct validity for this version of the LSRQ.

Learning Climate Questionnaire (LCQ).- -This questionnaire was adapted by Ref. [22] from the HealthCare Climate Questionnaire [55]. The LCQ measures the degree to which students perceive their instructors as autonomy supportive versus controlling. Autonomy support is described by the LCQ creators as the degree to which the instructor acknowledges the student's perspectives (e.g., "My DL instructor tries to understand how I see things before suggesting a new way to do things") and feelings (e.g., "I feel able to share my feelings with my DL instructor") and provides pertinent information and opportunities for choice while minimizing external pressures and demands (e.g., "My DL instructor listens to how I would like to do things") $[22,55]$. Acknowledging the student's perspectives and feelings is congruent with the "acknowledge negative feelings" instructor behavior described in Sec. II C, providing pertinent information and opportunities for choice is congruent with the "offer choices" behavior, and minimizing external pressures and demands is congruent with the "nurture inner motivational resources" behavior. The LCQ addressed the students' perceptions of their DL instructor (not the course lecturer). Students rated on a seven-point Likert-type scale their level of agreement or disagreement with each item. We did not include the first item of this 15-item questionnaire for reasons discussed in study II [9]. The total score for this measure is the sum of the 14 items and is our Instructor Autonomy Support (IAS) variable. The LCQ was administered only at T2 after the students had sufficient time to experience the degree to which their DL instructors were autonomy supportive. The LCQ has a single underlying factor and high internal consistency ([22], BD00). Cronbach's alpha for the present study was 0.92 .

These three measures of autonomy versus control are readily available for download at Ref. [56], after first registering and logging in to the Web site. This Web site also provides additional information about these measures, such as more detailed descriptions and information about their validity, reliability, and usage.

\section{Performance measures}

Grades.-Eight weekly quizzes were administered in lecture during weeks 2-9. The final exam was administered during the 11th (finals) week. Given that all T2 questionnaires were administered before the final exam, when controlling for grade at T2 in the analysis below, the quiz grade (hereafter Quizzes) was used. The quiz grade was determined by averaging quiz grades after dropping the quiz with the lowest score. The final course grade (hereafter Course Grade) out of 4.5 points (A $=3.5-4.5$, $\mathrm{B}=2.5-3.5$, etc.) was composed of quiz grades and the final exam. To control for academic ability (i.e., success in school in terms of performance) in our analysis we used incoming grade point average (hereafter GPA). GPA was given by total grade points divided by total units attempted, where grade points were assigned for each unit on a 4-point scale $(\mathrm{A}+$ and $\mathrm{A}=4.0, \mathrm{~A}-=3.7, \mathrm{~B}+=3.3, \mathrm{~B}=3.0$, etc.). Table I provides a summary description of the key variables of this work.

\section{ANALYSIS AND FINDINGS}

In this section, we first describe our analysis generally and present summary statistics of key variables (mean values, correlation matrix, etc.). We then present our tests of each of the four hypotheses of this study in turn.

For our analysis we used the Stata statistical software. To compare distributions of dependent variables with interval scales (i.e., Course Grade, Quizzes, and GPA), we used parametric statistics, specifically $t$ tests. All $t$ tests were two sided and for unpaired samples (i.e., when comparing different students) did not assume the two distributions being compared had the same variance; thus, Satterthwaite's degrees of freedom [57] were reported. To compare distributions of dependent variables with ordinal scales (i.e., variables from measures with Likerttype scales), we used nonparametric statistical tests. Specifically, we used the Wilcoxon-Mann-Whitney test to compare two unpaired samples, the Kruskal-Wallis test to compare more than two unpaired samples, and the Wilcoxon signed-rank test to compare two paired samples.

We performed hierarchical regression (not to be confused with hierarchical linear modeling) at various points in our analysis, meaning that we performed a multiple linear regression in steps, adding independent variables at each step. This was done to test whether the key predictor 
TABLE I. A summary description of key variables in this study. Variables are grouped by measure type. Also shown are variable name, either a description of the variable or an example item from the corresponding measure, and when the corresponding measure was administered to students.

\begin{tabular}{ll}
\hline \hline Variable & Description or example item \\
\hline Adjustment & Administered \\
Interest/Enjoyment & "I enjoy learning about introductory physics" \\
Perceived Competence & "I have confidence in my ability to be successful in introductory physics", \\
Anxiety & Measures amount of anxiety students feel about taking introductory physics \\
Grade Orientation & "I care much more about what I learn than the grades I get" (reverse scored) \\
Autonomy versus control & Measures degree to which students' reasons for studying physics are autonomous \\
Relative Autonomy & versus controlled (i.e., because they want to versus have to) \\
Index (RAI) & Measures perceptions of students about degree to which their DL instructor is \\
Instructor Autonomy & autonomy supportive versus controlling \\
Support (IAS) & Autonomous (GCOS-A), controlled (GCOS-C), and impersonal (GCOS-I) general \\
General Causality & motivational orientations \\
Orientations & \\
Subscales (GCOS) & \\
Performance & Incoming grade point average \\
GPA & Grade at T2, just before the final exam \\
Quizzes & Final grade in the course \\
Course Grade & Dummy variable for gender; 0 for males and 1 for females \\
Other: & Dummy variables for the different instructors; e.g., Instructor 1 has a value of 1 for \\
Female & the students of instructor 1 and 0 otherwise \\
Instructor 1, &
\end{tabular}

variable(s) accounted for a statistically significant amount of the variance in the dependent variable above and beyond the control variables. This was done by testing whether the change in the R-squared of the model upon taking the step in which the key predictor variable (s) was added was significantly different from zero. The quoted standardized correlation coefficients ( $\beta$ 's) in Tables IV-VII are those of the full model once all steps were taken. Given that a standardized dummy variable has little meaning, we also quoted the correlation coefficient for dummy independent variables when only the dependent variable was standardized, which we refer to as $\beta_{\mathrm{DV}}$.

For each model used to test our hypotheses, we investigated whether the data met the assumptions of multiple linear regression as follows. We investigated whether the dependent variables could be described as a linear function of the key predictor variables by visually inspecting component-plus-residual plots after smoothing the data (e.g., with a median trace). We found that linear models fit the data quite well (see, e.g., Figs. 2 and 3). We also searched for outliers that may heavily influence the results of our regression models. We ran the models with and without these data and found that our main results did not change. Finally, we estimated the magnitude of multicollinearity (i.e., the interdependence of independent variables) in our models with the variance inflation factor, which gives the factor by which the variance of each regression coefficient is increased because of multicollinearity. Common rules of thumb are that multicollinearity may be high and warrant further investigation for inflation factors above 5 or 10 . We found all factors to be below 2 (with an average of around 1.3), suggesting low multicollinearity. Additionally, we used robust standard errors, which does not assume homoscedasticity of the residuals (i.e., that the variance of the residuals is the same for all values of the independent variables), potentially yielding better correlation coefficient standard errors.

The mean values of key variables at $\mathrm{T} 1$ and $\mathrm{T} 2$ are shown in Table II. The $z$ statistics for tests comparing T1 and T2 values are also given. On average, students became significantly less interested in learning physics and came to study physics for less autonomous reasons. On a more positive note, on average, students' anxiety about taking physics decreased significantly.

Next, we show IAS for individual instructors in the box plot of Fig. 1. Instructor 5 is included in Fig. 1 for comparison only and is not included in any analysis, as described above. We compared IAS among the instructors using the Kruskal-Wallis test to get an idea of whether IAS, as a student-perceived autonomy support, is measuring an actual instructor effect. We found a statistically significant difference in IAS among instructors 1, 2, 3, 4, and 6 $\left[\chi^{2}(4, N=182)=18.8, \quad p<0.001\right], \quad$ suggesting that IAS is to some extent measuring an instructor effect. This difference is mostly due to instructor 4 having a lower IAS than the other instructors combined $\left[\chi^{2}(1, N=182)=13.6, p<0.001\right]$ with a Cohen's $d$ of 
TABLE II. Mean values of key variables measured at the beginning of the course (T1) and end of the course (T2) for all students combined $(N=182)$, Cohen's $d$ and 95\% confidence interval for T2 minus T1, and Wilcoxon signed-rank test $z$ statistic for T2 versus $\mathrm{T} 1\left(^{*} p<0.05,{ }^{* *} p<0.01\right.$, and $\left.{ }^{* * *} p<0.001\right)$. We see that on average students became less interested in learning physics, became less anxious about studying physics, and came to study physics for less autonomous reasons over the duration of the course.

\begin{tabular}{lcccc}
\hline \hline & T1 & T2 & T1 versus T2 & T1 versus T2 \\
Variable & Mean (SD) & Mean (SD) & $d$ CI] & $-2.90^{* *}$ \\
\hline Interest/Enjoyment & $28.8(6.9)$ & $27.1(7.2)$ & $-0.24[-0.44,-0.03]$ & 1.74 \\
Perceived Competence & $18.2(3.6)$ & $18.6(3.8)$ & $0.10[-0.11,0.31]$ & $-3.54^{* * *}$ \\
Anxiety & $33.6(9.3)$ & $31.4(9.2)$ & $-0.24[-0.45,-0.03]$ & -0.18 \\
Grade Orientation & $15.9(2.7)$ & $15.8(2.9)$ & $-0.04[-0.24,0.17]$ & $-3.14^{* *}$ \\
Autonomous Reasons & $18.2(3.3)$ & $17.1(3.6)$ & $-0.30[-0.51,-0.09]$ & -0.56 \\
Controlled Reasons & $24.0(4.1)$ & $23.7(4.7)$ & $-0.06[-0.26,0.15]$ & \\
Instructor Autonomy Support & & $80.6(11.9)$ & &
\end{tabular}

$0.71(95 \%$ CI $[0.36,1.06])$, a large to medium-size effect according to Cohen's guidelines [58].

As there was a statistically significant difference among instructors in instructor autonomy support as perceived by the students (IAS), and we expected instructor autonomy support to be correlated with our dependent variables, we accounted for any potential differences among instructors in the dependent variables of the models we used to test our hypotheses. These dependent variables are the adjustment and performance variables as well as autonomous reasons for studying physics (RAI). We accounted for instructor differences in these variables by including in the regression models the instructor variables: Instructor 1 , Instructor 2,

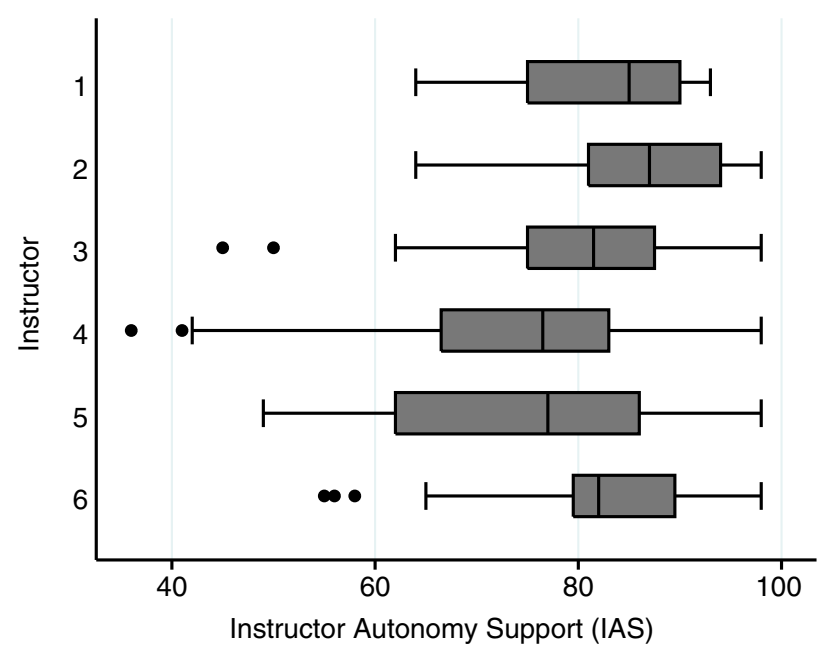

FIG. 1. Box plots for individual instructors comparing the level of instructor autonomy support as perceived by the students in their discussion/lab sections. Instructor 5 is included here for comparison only, and is not included elsewhere in this work. Box plots give the five-number summary: median (vertical line in the box), 25th and 75th percentile (edges of the box), lower and upper adjacent values (i.e, the lowest datum and highest datum within 1.5 interquartile range of the lower and upper quartile, respectively; ends of the whiskers), and values outside the adjacent values (dots). We see that instructor 4 has a lower IAS than Instructors 1, 2, 3, and 6, suggesting that student-perceived IAS is measuring an instructor effect.
Instructor 3, and Instructor 4. The instructor variables were defined to be 1 for students of that instructor and 0 otherwise. An Instructor 6 variable need not be included since a value of zero for all other instructors corresponds to instructor 6.

The correlations between the key variables of this study are presented in Table III (with beta coefficients and significance levels) and are used to guide our analysis as described below. We now test each of our four hypotheses in turn.

\section{A. Tests of hypothesis 1}

We tested whether relatively more autonomous reasons for studying physics when entering the course [RAI at T1, hereafter RAI (T1)] predicted a more positive experience in the course, as measured by T2 adjustment score. In step 1 of our hierarchical regression model, we regressed the T2 adjustment score onto the control variables, namely, Quizzes to control for grade at T2, each GCOS subscale to control for general motivational orientations and thus isolate course-specific autonomous motivation, and Female to control for differences by gender. In step 2 we controlled for any differences among instructors, with instructor 6 as the reference category. In step 3 we regressed the T2 adjustment score onto the key predictor variable, RAI (T1). The full model is thus

$$
\begin{aligned}
\operatorname{score}(\mathrm{T} 2)= & B_{0}+B_{1} \text { Quizzes }+B_{2} \mathrm{GCOS}-A \\
& +B_{3} \text { GCOS- } C+B_{4} \mathrm{GCOS}-I+B_{5} \text { Female } \\
& +B_{6} \text { Instructor } 1+B_{7} \text { Instructor } 2 \\
& +B_{8} \text { Instructor } 3+B_{9} \text { Instructor } 4 \\
& +B_{10} \mathrm{RAI}(\mathrm{T} 1) .
\end{aligned}
$$

As seen in Table IV, the standardized correlation coefficient $(\beta)$ of RAI (T1) was significant for Interest/Enjoyment at T2 $[\beta(10,171)=0.26,95 \%$ CI $[0.09,0.43]]$ with a medium effect size [58] and Grade Orientation at T2 $[\beta(10,171)=-0.22,[-0.39,-0.04]]$ with a small to medium effect size. The change in $R^{2}$ upon adding RAI 
TABLE III. Pairwise correlation matrix $(N=182)$ showing the standardized correlation coefficients $(\beta$ 's $)$ of key variables with accompanying $p$ values, where ${ }^{*} p<0.05,{ }^{* *} p<0.01,{ }^{* * *} p<0.001$.

\begin{tabular}{|c|c|c|c|c|c|c|c|c|c|c|c|c|c|}
\hline & (1) & (2) & (3) & (4) & (5) & (6) & (7) & (8) & (9) & (10) & (11) & (12) & (13) \\
\hline (1) IAS & 1 & & & & & & & & & & & & \\
\hline (2) GCOS-A & $0.15^{*}$ & 1 & & & & & & & & & & & \\
\hline (3) GCOS-C & 0.01 & $0.42^{* * *}$ & 1 & & & & & & & & & & \\
\hline (4) GCOS-I & -0.06 & 0.06 & $0.16^{*}$ & 1 & & & & & & & & & \\
\hline (5) RAI (T1) & 0.02 & 0.05 & $-0.15^{*}$ & $-0.26^{* * *}$ & 1 & & & & & & & & \\
\hline (6) RAI (T2) & $0.15^{*}$ & -0.04 & $-0.16^{*}$ & -0.11 & $0.43^{* * *}$ & 1 & & & & & & & \\
\hline $\begin{array}{l}\text { (7) Interest/ } \\
\text { Enjoyment (T2) }\end{array}$ & $0.29^{* * *}$ & -0.01 & -0.08 & $-0.17^{*}$ & $0.29^{* * *}$ & $0.50^{* * *}$ & 1 & & & & & & \\
\hline $\begin{array}{l}\text { (8) Perceived } \\
\text { Competence (T2) }\end{array}$ & 0.14 & 0.09 & -0.09 & $-0.16^{*}$ & $0.18^{*}$ & $0.32^{* * *}$ & $0.46^{* * *}$ & 1 & & & & & \\
\hline (9) Anxiety (T2) & $-0.23^{* *}$ & 0.00 & $0.15^{*}$ & $0.30^{* * *}$ & $-0.19 *$ & $-0.38^{* * *}$ & $-0.47^{* * *}$ & $-0.65^{* * *}$ & 1 & & & & \\
\hline $\begin{array}{l}\text { (10) Grade } \\
\text { Orientation (T2) }\end{array}$ & 0.09 & $0.18^{*}$ & $0.23^{* *}$ & 0.09 & $-0.21^{* *}$ & $-0.38^{* * *}$ & $-0.25^{* * *}$ & -0.02 & $0.21^{* *}$ & 1 & & & \\
\hline (11) Quizzes & $0.15^{*}$ & 0.03 & -0.07 & -0.05 & 0.06 & 0.10 & $0.25^{* * *}$ & $0.49^{* * *}$ & $-0.38^{* * *}$ & 0.04 & 1 & & \\
\hline (12) Course Grade & $0.18^{*}$ & 0.04 & -0.08 & -0.06 & 0.04 & 0.14 & $0.27^{* * *}$ & $0.49^{* * *}$ & $-0.41^{* * *}$ & 0.00 & $0.87^{* * *}$ & 1 & \\
\hline (13) Female & 0.08 & 0.10 & -0.08 & -0.01 & 0.03 & -0.13 & $-0.28^{* * *}$ & $-0.21^{* *}$ & $0.19^{*}$ & $0.18^{*}$ & $-0.20^{* *}$ & $-0.19^{*}$ & 1 \\
\hline
\end{tabular}

(T1) as a predictor variable in step 2 was also significant for these two dependent variables. Thus, RAI (T1) predicted higher Interest/Enjoyment at T2 and lower Grade Orientation at T2, substantiating hypothesis 1 for these two adjustment variables. We found no such correlation for Perceived Competence $[\beta(10,171)=0.11,[-0.02,0.24]]$ or Anxiety $[\beta(10,171)=-0.09,[-0.22,0.03]]$ at $\mathrm{T} 2$. We note that the fact that women had a less positive experience in the course as measured by all four T2 adjustment scores, particularly, Interest/Enjoyment, is investigated further in study II [9]. Finally, we note that the change in $R^{2}$ for step 2 was not statistically significant, so the model did not improve significantly upon controlling for the effects of the different instructors on the T2 adjustment score.

\section{B. Tests of hypothesis 2}

We tested whether more positive changes in adjustment and relative autonomous reasons for studying physics were positively correlated with how autonomy supportive the students perceived their instructors to be. In step 1 we regressed either the $\mathrm{T} 2$ adjustment score or the RAI score onto the $\mathrm{T} 1$ score (to create a change score) and onto

TABLE IV. Regressions of each dependent variable (Interest/Enjoyment, Perceived Competence, Anxiety, and Grade Orientation) at the end of the course (T2) onto control variables (steps 1 and 2) and initial relative autonomous reasons for studying physics [RAI (T1), step 3]. Standardized correlation coefficients $\left(\beta\right.$ 's) are given with accompanying $p$ values, where ${ }^{*} p<0.05,{ }^{* *} p<0.01,{ }^{* * *} p<0.001$. Given that fully standardized coefficients have little meaning for dummy variables, the coefficients when only the dependent variables were standardized ( $\beta_{\mathrm{DV}}$ 's) are given in parentheses for dummy independent variables (i.e., Female and the instructor variables). The degrees of freedom, $R^{2}$ changes, and $R^{2}$ of the full model are also given for each model. We see that more autonomous reasons for studying physics when entering the course predicted significantly higher interest and enjoyment in learning physics and less of a focus on grades (versus learning) $(N=182)$.

\begin{tabular}{llcccc}
\hline \hline & & Interest/Enjoyment (T2) & Perceived Competence (T2) & Anxiety (T2) & Grade Orientation (T2) \\
\hline Step 1 & Quizzes & $0.18^{*}$ & $0.45^{* * *}$ & $-0.35^{* * *}$ & 0.08 \\
& GCOS-A & 0.02 & 0.12 & -0.06 & 0.07 \\
& GCOS-C & -0.04 & -0.08 & 0.11 & $0.20^{*}$ \\
& GCOS-I & -0.08 & -0.11 & $0.26^{* * *}$ & 0.02 \\
& Female & $-0.25^{* * *}(-0.51)$ & $0.13^{*}(-0.28)$ & $0.13^{*}(0.26)$ & $0.20^{*}(0.40)$ \\
Step 2 & Instructor 1 & $-0.08(-0.26)$ & $0.01(0.03)$ & $0.01(0.02)$ & $0.16^{* *}(0.53)$ \\
& Instructor 2 & $0.01(0.02)$ & $-0.10(-0.26)$ & $0.13(0.32)$ & $0.08(0.19)$ \\
& Instructor 3 & $-0.05(-0.12)$ & $-0.07(-0.16)$ & $-0.03(-0.06)$ & $-0.07(-0.17)$ \\
& Instructor 4 & $0.02(0.04)$ & $0.02(0.05)$ & $-0.07(-0.16)$ & $-0.09(-0.21)$ \\
Step 3 & RAI (T1) & $0.26^{* *}$ & 0.11 & -0.09 & $-0.22^{*}$ \\
& Degrees of freedom & $(10,171)$ & $(10,171)$ & $(10,171)$ & $(10,171)$ \\
& $R^{2}$ Change for step 2 & 0.01 & 0.01 & 0.03 & 0.04 \\
& $R^{2}$ Change for step 3 & $0.06^{* * *}$ & 0.01 & 0.01 & $0.04^{* *}$ \\
& $R^{2}$ of full model & $0.21^{* * *}$ & $0.31^{* * *}$ & $0.29^{* * *}$ & $0.19^{* * *}$ \\
\hline \hline
\end{tabular}


Female. In step 2 we controlled for any differences among instructors by including the instructor variables. In step 3 we regressed onto the key predictor variable, IAS. Thus, the full model is

$$
\begin{aligned}
\operatorname{score}(T 2)= & B_{0}+B_{1} \operatorname{score}(T 1)+B_{2} \text { Female } \\
& +B_{3} \text { Instructor } 1+B_{4} \text { Instructor } 2 \\
& +B_{5} \text { Instructor } 3+B_{6} \text { Instructor } 4 \\
& +B_{7} \text { IAS. }
\end{aligned}
$$

It is important to note that including the instructor variables in this multiple regression model (and the others in our analysis) controls both the dependent variable [59] and the other independent variables (e.g., IAS) for differences among instructors. Thus $B_{7}$, for example, is the correlation of within-instructor differences in IAS with withininstructor differences in change in adjustment (after also controlling for other independent variables, Female in this case).

As seen in Table V, we found IAS to be significantly positively correlated with changes in Interest/Enjoyment $[\beta(7,174)=0.31,95 \%$ CI $[0.19,0.44]]$ with a medium effect size, changes in RAI $[\beta(7,174)=0.24,[0.12,0.36]]$ with a small to medium effect size, and changes in Perceived Competence $[\beta(7,174)=0.16,[0.02,0.29]]$ with a small effect size. IAS was also significantly negatively correlated with changes in Anxiety $[\beta(7,174)=-0.23, \quad[-0.35,-0.11]]$ with a small to medium effect size. IAS was not correlated with changes in Grade Orientation $[\beta(7,174)=0.08,[-0.03,0.18]]$.
Thus, all of the above correlations that were statistically significant were in the expected direction.

Component-plus-residual plots for the models with Interest/Enjoyment (T2), Anxiety (T2), and RAI (T2) as dependent variables are shown in Fig. 2. For each of these dependent variables, the lines in Fig. 2 are $B_{0}+B_{7}$ IAS and thus have slope $B_{7}$ that shows the dependence of the dependent variable on IAS after controlling for the other variables in the model. The data points are the residuals of the full model plus the $B_{0}$ and $B_{7}$ IAS components of the model; thus, the line is the best linear fit to the data points. The variance of data points in a component-plus-residual plot will, however, be less than the actual variance if the independent variable in the plot is highly correlated with other independent variables in the model, which is a small effect here [60].

As seen in Table III, Interest/Enjoyment, Perceived Competence, and Anxiety at T2 depended significantly on Quizzes (i.e., the student's grade at T2). Therefore, we also performed the regressions of Eq. (2) for these three adjustment variables while also controlling for Quizzes. The correlations for Interest/Enjoyment $[\beta(8,173)=0.29$, $95 \%$ CI $[0.17,0.42]]$ and Anxiety $[\beta(8,173)=-0.21$, $[-0.32,-0.10]]$ remained statistically significant and that of Perceived Competence, which of the three had the largest beta for Quizzes, became statistically insignificant $[\beta(8,173)=0.12,[-0.01,0.25]]$. As will be seen below (hypothesis 3), IAS correlated directly with performance (at least for a subset of students). The fact that the correlations remained for Interest/Enjoyment and Anxiety when controlling for Quizzes showed that IAS correlated directly with changes in Interest/Enjoyment and Anxiety even after

TABLE V. Regressions of each dependent variable (Interest/Enjoyment, Perceived Competence, Anxiety, Grade Orientation, and relative autonomous reasons for studying physics) at the end of the course (T2) onto the score at the beginning of the course to create a change score and Female (step 1), instructor variables (step 2), and instructor autonomy support as perceived by the students (step 3). Standardized correlation coefficients ( $\beta$ 's) are given with accompanying $p$ values, where ${ }^{*} p<0.05,{ }^{* *} p<0.01$, ${ }^{* * *} p<0.001$. For the dummy independent variables (Female and the instructor variables), the coefficients when only the dependent variables were standardized ( $\beta_{\mathrm{DV}}$ 's) are also given in parenthesis. The degrees of freedom, $R^{2}$ changes, and $R^{2}$ of the full model are also given for each model. We see that students who perceived their instructors as more autonomy supportive tended to become significantly more interested in learning physics and less anxious about taking physics and came to study physics for more autonomous reasons (i.e., more because they wanted to versus had to) $(N=182)$.

\begin{tabular}{llccccc}
\hline \hline & & $\begin{array}{c}\text { Interest/Enjoyment } \\
(\mathrm{T} 2)\end{array}$ & $\begin{array}{c}\text { Perceived Competence } \\
(\mathrm{T} 2)\end{array}$ & $\begin{array}{c}\text { Anxiety } \\
(\mathrm{T} 2)\end{array}$ & $\begin{array}{c}\text { Grade Orientation } \\
(\mathrm{T} 2)\end{array}$ & $\begin{array}{c}\text { RAI } \\
(\mathrm{T} 2)\end{array}$ \\
\hline Step 1 & T1 score & $0.52^{* * *}$ & $0.58^{* * *}$ & $0.60^{* * *}$ & $0.65^{* * *}$ & $0.45^{* * *}$ \\
& Female & $-0.19^{* * *}(-0.40)$ & $-0.04(-0.08)$ & $-0.03(-0.06)$ & $0.12^{*}(0.24)$ & $-0.13^{*}(-0.26)$ \\
Step 2 & Instructor 1 & $-0.11(-0.37)$ & $-0.00(-0.00)$ & $-0.00(-0.01)$ & $0.09(0.29)$ & $-0.15(-0.50)$ \\
& Instructor 2 & $-0.03(-0.07)$ & $-0.05(-0.11)$ & $0.05(0.13)$ & $0.05(0.13)$ & $-0.22^{* *}(-0.54)$ \\
& Instructor 3 & $-0.06(-0.14)$ & $-0.04(-0.10)$ & $-0.03(-0.07)$ & $-0.07(-0.16)$ & $-0.03(-0.07)$ \\
& Instructor 4 & $0.07(0.17)$ & $0.03(0.06)$ & $-0.12(-0.28)$ & $-0.06(-0.13)$ & $0.11(0.25)$ \\
Step 3 & IAS & $0.31^{* * *}$ & $0.16^{*}$ & $-0.23^{* *}$ & 0.08 & $0.24^{* * *}$ \\
& Degrees of freedom & $(7,174)$ & $(7,174)$ & $(7,174)$ & $(7,174)$ & $(7,174)$ \\
& $R^{2}$ Change for step 2 & 0.01 & 0.00 & 0.00 & 0.03 & $0.05^{*}$ \\
& $R^{2}$ Change for step 3 & $0.09^{* * *}$ & $0.02^{*}$ & $0.05^{* * *}$ & 0.01 & $0.05^{*}$ \\
& $R^{2}$ of full model & $0.44^{* * *}$ & $0.39^{* * *}$ & $0.43^{* * *}$ & $0.49^{* * *}$ & $0.30^{* * *}$ \\
\hline \hline
\end{tabular}



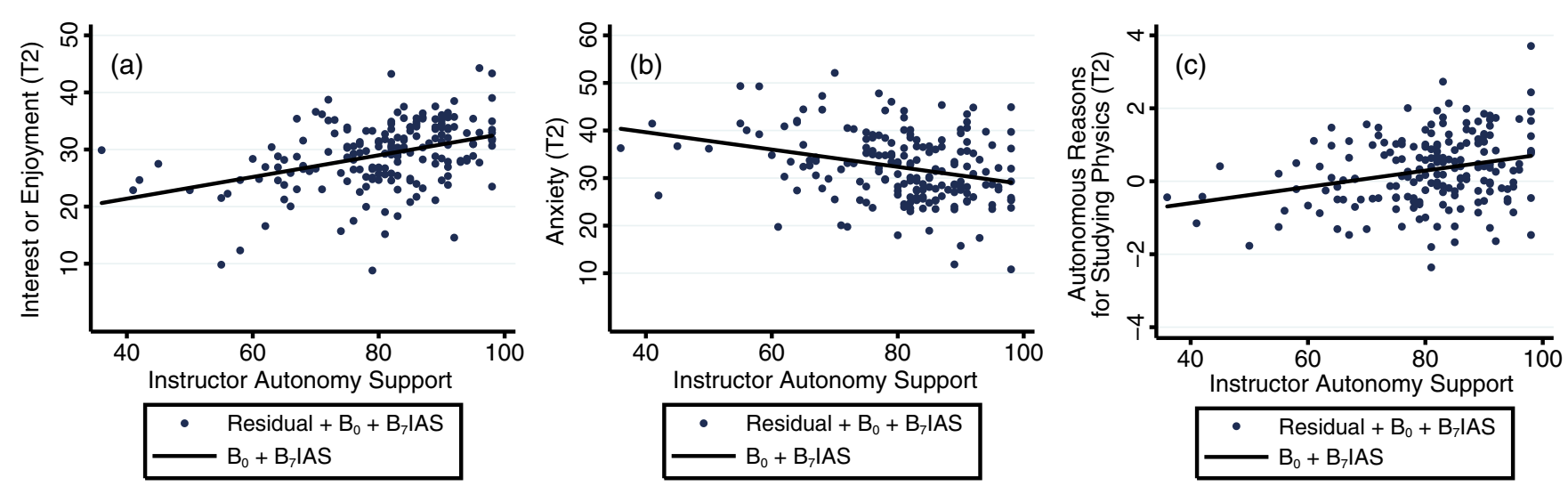

FIG. 2. Component-plus-residual plots for the models of Table V that had (a) Interest/Enjoyment, (b) Anxiety, and (c) relative autonomous reasons for studying physics at the end of the course (T2) as dependent variables, as these were the dependent variables in Table V that depended most significantly on instructor autonomy support as perceived by the students. The slope of the line in each plot is $B_{7}$, the best-fit coefficient of IAS from the multiple regression and thus shows the dependence of the dependent variable on IAS after controlling for the other variables in the model. It is also the best linear fit to the data points, which are the residuals of the full model plus the $B_{0}$ and $B_{7}$ IAS components of the model. The plots show that students who perceived their instructors as more autonomy supportive tended to become significantly more interested in learning physics and less anxious about taking physics and came to study physics for more autonomous reasons (i.e., more because they wanted to versus had to).

taking into account the correlations of these variables and IAS with performance.

Additionally, we tested whether the correlation of changes in adjustment with IAS was moderated by students' initial relative autonomous reasons for studying physics by including RAI (T1) and the interaction term IAS $\times$ RAI (T1) as independent variables in the models for the four adjustment variables. We found no statistically significant correlations for these two variables, suggesting that the correlation of changes in adjustment with IAS was not significantly different for those taking the course for relatively more or less autonomous reasons.

The above results substantiate hypothesis 2 . We note that the significant dependence of changes in Interest/ Enjoyment on gender, with a small to medium effect size, is investigated further in study II [9].

Finally, as seen in Table V, including instructor differences in the model in step 2 only improved the model significantly for the RAI model (change in $R^{2}=0.05$, $p=0.022)$ and marginally so for the Grade Orientation model (change in $R^{2}=0.03, p=0.057$ ). It could be that these instructor differences were entirely due to differences in IAS between instructors and the resulting differences in the dependent variables from the correlation of IAS with the dependent variables. If this were the case, and we controlled for IAS before including the instructor variables, then the change in $R^{2}$ upon adding the instructor variables in the last step would be zero. We tested this by simply running the same model as before but with steps 2 and 3 switched, thus controlling for IAS before including the instructor variables. We found a change in $R^{2}$ of 0.08 $(p=0.001)$ for the RAI model and $0.02(p=0.14)$ for the
Grade Orientation model. Thus, differences in changes in RAI among instructors accounted for a statistically significant amount of the variance in changes in RAI, above and beyond gender effects and the correlation of changes in RAI with IAS, suggesting that instructors had a statistically significant effect on changes in relative autonomous reasons for studying physics that was not attributable to IAS.

\section{Tests of hypothesis 3}

We first tested whether performance in the course was positively correlated with how autonomy supportive the students perceived their instructor to be. We regressed Course Grade onto GPA, Female, the instructor variables, and IAS in the model

$$
\begin{aligned}
\text { Course Grade }= & B_{0}+B_{1} \text { GPA }+B_{2} \text { Female } \\
& +B_{3} \text { Instructor } 1+B_{4} \text { Instructor } 2 \\
& +B_{5} \text { Instructor } 3+B_{6} \text { Instructor } 4 \\
& +B_{7} \text { IAS. }
\end{aligned}
$$

We found the correlation of Course Grade with IAS to be statistically insignificant $[\beta(7,154)=0.12,95 \%$ CI $[-0.01,0.25]]$. We then tested whether this correlation was moderated by the students' initial relative autonomous reasons for studying physics. We regressed Course Grade onto GPA, Female, the instructor variables, IAS, RAI (T1), and the interaction term $\mathrm{RAI}(\mathrm{T} 1) \times \mathrm{IAS}$. As seen in the first column of numerical data in Table VI, we found that Course Grade correlated positively with the interaction 
TABLE VI. Regression testing for the dependence of Course Grade on Instructor Autonomy Support, relative autonomous reasons for studying physics at the beginning of the course [RAI (T1)], and the interaction of IAS and RAI (T1), after controlling for GPA, gender, and differences in student performance among instructors (first column of numerical data). Regressions clarifying the interaction of IAS and RAI (T1) are also shown, one for students with below average RAI (T1) (penultimate column) and one for students with above average RAI (T1) (last column). Standardized correlation coefficients ( $\beta$ 's) are given with accompanying $p$ values, where ${ }^{*} p<0.05$, ${ }^{* *} p<0.01,{ }^{* * *} p<0.001$. For the dummy variables (Female and the instructor variables), the coefficients when only the dependent variables were standardized ( $\beta_{\mathrm{DV}}$ 's) are given in parentheses. The degrees of freedom, $R^{2}$ changes, and $R^{2}$ of the full model are also given for each model. We see that the correlation of performance with IAS depended significantly on students' initial relative autonomous reasons for studying physics. IAS was positively correlated with performance, only for students initially studying physics for relatively autonomous reasons.

\begin{tabular}{|c|c|c|c|c|}
\hline & & Course Grade & $\begin{array}{l}\text { Course Grade } \\
\text { Low RAI (T1) }\end{array}$ & $\begin{array}{c}\text { Course Grade } \\
\text { High RAI (T1) }\end{array}$ \\
\hline \multirow[t]{2}{*}{ Step 1} & GPA & $0.58^{* * *}$ & $0.61^{* * *}$ & $0.53^{* * *}$ \\
\hline & Female & $-0.27^{* * *}(-0.54)$ & $-0.24^{*}(-0.49)$ & $-0.28^{* *}(-0.56)$ \\
\hline \multirow[t]{4}{*}{ Step 2} & Instructor 1 & $-0.12^{*}(-0.40)$ & $-0.14^{*}(-0.50)$ & $-0.03(-0.09)$ \\
\hline & Instructor 2 & $-0.19^{*}(-0.46)$ & $-0.26^{* *}(-0.64)$ & $-0.06(-0.15)$ \\
\hline & Instructor 3 & $-0.18^{*}(-0.45)$ & $-0.26^{*}(-0.65)$ & $-0.07(-0.17)$ \\
\hline & Instructor 4 & $-0.25^{* * *}(-0.57)$ & $-0.33^{* * *}(-0.76)$ & $-0.01(-0.23)$ \\
\hline \multirow[t]{8}{*}{ Step 3} & RAI (T1) & 0.10 & & \\
\hline & IAS & 0.09 & -0.03 & $0.25^{* *}$ \\
\hline & $\operatorname{RAI}(\mathrm{T} 1) \times \mathrm{IAS}$ & $0.13^{*}$ & & \\
\hline & $N$ & 162 & 87 & 75 \\
\hline & Degrees of freedom & $(9,152)$ & $(7,79)$ & $(7,67)$ \\
\hline & $R^{2}$ change for step 2 & $0.05^{*}$ & $0.09^{*}$ & 0.03 \\
\hline & $R^{2}$ change for step 3 & $0.04^{*}$ & 0.00 & $0.05^{*}$ \\
\hline & $R^{2}$ of full model & $0.42^{* * *}$ & $0.47^{* * *}$ & $0.39^{* * *}$ \\
\hline
\end{tabular}

term $\operatorname{RAI}(\mathrm{T} 1) \times \operatorname{IAS}[\beta(9,152)=0.13,[0.004,0.25]]$. We investigated this interaction by running the model in Eq. (3) individually for the subset of students initially relatively low in autonomous reasons for studying physics [i.e., $\operatorname{RAI}(\mathrm{T} 1)<0$, corresponding to less than the mean RAI (T1)] and the subset initially relatively high $[\operatorname{RAI}(\mathrm{T} 1)>0]$. We can loosely interpret those with low RAI (T1) as studying physics more because they "had" to and those with high RAI (T1) as studying physics more because they "wanted" to. As seen in Table VI, Course Grade was positively correlated with IAS only for the subset of students initially high in RAI $[\beta(7,67)=0.25$, $[0.06,0.43]]$ with a medium size effect, not for students initially low in RAI $[\beta(7,79)=-0.03,[-0.22,0.17]]$. Component-plus-residual plots for these two subsets of students are shown in Fig. 3. The lines in Fig. 3 are $B_{0}+$ $B_{3}$ IAS and thus have slope $B_{3}$ that shows the dependence of Course Grade on IAS after controlling for the other variables in the model. The data points are the residuals of the full model plus the $B_{0}$ and $B_{3}$ IAS components of the model; thus, the line is the best linear fit to the data points. For the high RAI subset of students, the variance of the data points in the component-plus-residual plot is very similar to that of the actual variance, and the correlation of performance with IAS remained upon removing the three points in Fig. 3 with the lowest IAS values $[\beta(7,64)=0.20$, $[0.002,0.40]]$.
These findings substantiate the hypothesis that the correlation of performance with instructor autonomy support would be moderated by initial relative autonomous reasons for studying physics. While in this study the correlation was stronger for those initially relatively high in autonomous reasons for studying physics, in BD00 it was stronger for those initially relatively low.

Finally, as seen in Table VI, including differences among instructors in step 2 improved the model by a statistically significant amount for all students (change in $R^{2}=0.05$, $\mathrm{p}=0.012$ ) and for those relatively low in autonomous reasons for studying physics (change in $R^{2}=0.09$, $\mathrm{p}=0.012$ ), but not for those relatively high (change in $R^{2}=0.03, \mathrm{p}=0.61$ ). To determine whether this effect was due to differences in IAS among instructors and the resulting differences in performance from the correlation of performance with IAS, we ran the same models as in Table VI but with steps 2 and 3 switched so as to control for IAS before including the instructor variables. We found a statistically significant change in $R^{2}$ upon including the instructor variables of $0.04(p=0.03)$ for all students and of $0.09(p=0.012)$ for those with low RAI (T1). The change in $R^{2}$ was not statistically significant for those with high RAI (T1) (change in $R^{2}=0.01, p=0.96$ ). Thus, differences among instructors in student performance accounted for a statistically significant amount of the variance in student performance, above and beyond 

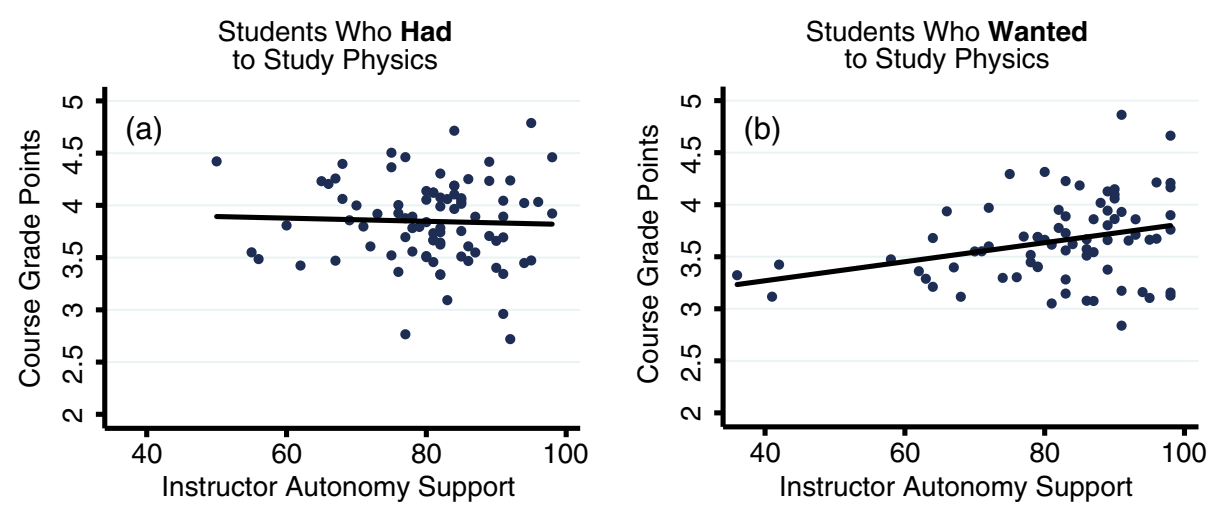

$\begin{array}{ll}- & \text { Residual }+B_{0}+B_{3} I A S \\ & B_{0}+B_{3} I A S\end{array}$

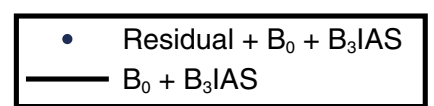

FIG. 3. Component-plus-residual plots for the two models of Table VI that were for (a) the subset of students initially taking physics more because they had to [i.e., for relatively less autonomous reasons or RAI $(\mathrm{T} 1)<0$ ] and for (b) the subset initially taking physics more because they wanted to [i.e., for relatively more autonomous reasons or RAI (T1) $>0$ ]. The slope of the line in each plot is $B_{3}$, the best-fit coefficient of IAS from the multiple regression, and thus shows the dependence of Course Grade on IAS after controlling for incoming GPA, gender, and differences among instructors in student performance. It is also the best linear fit to the data points, which are the residuals of the full model plus the $B_{0}$ and $B_{3}$ IAS components of the model. These plots show the significantly different correlation of Instructor Autonomy Support with performance depending on students' initial relative autonomous reasons for studying physics. IAS was positively correlated with performance, only for students initially studying physics for relatively autonomous reasons.

incoming GPA, gender, and IAS for those students initially relatively low in autonomous reasons for studying physics, suggesting that instructors had a statistically significant effect on the performance of these students that was not attributable to IAS.

\section{Tests of hypothesis 4}

We tested the hypothesis that students who came to study physics for relatively more autonomous reasons over the duration of the quarter would tend to perform better. To do this, we first created a RAI Change Score variable from the residual of the regression of RAI (T2) onto RAI (T1). We then regressed Course Grade onto GPA to control for academic ability, Female to control for gender effects, the instructor variables to control for differences in student performance among instructors, and RAI Change Score. Thus, our model was

$$
\begin{aligned}
\text { Course Grade }= & B_{0}+B_{1} \text { GPA }+B_{2} \text { Female } \\
& +B_{3} \text { Instructor } 1+B_{4} \text { Instructor } 2 \\
& +B_{5} \text { Instructor } 3+B_{6} \text { Instructor } 4 \\
& +B_{7} \text { RAI Change Score. }
\end{aligned}
$$

As seen in Table VII, we found a statistically significant positive correlation between performance and RAI Change Score $[\beta(7,154)=0.17,95 \%$ CI $[0.04,0.31]]$ with a small to medium size effect, providing support for hypothesis 4 . This correlation is investigated further in study II [9].

We also tested whether this correlation was entirely due to the correlations of IAS with both performance (hypothesis 3, at least for a subset of students) and change in RAI (hypothesis 2) that were found above. We did this by adding IAS as a control variable in the above model. Upon doing so, we found that the correlation of Course Grade with RAI Change Score remained

TABLE VII. Regression testing for the dependence of Course Grade on change over the quarter in relative autonomous reasons for studying physics (RAI Change Score), after controlling for GPA and Female (step 1) and instructor variables (step 2). Standardized correlation coefficients $(\beta$ 's) are given with accompanying $p$ values, where ${ }^{*} p<0.05,{ }^{* *} p<0.01,{ }^{* * *} p<0.001$. For the dummy independent variables (Female and the instructor variables), the coefficients when only the dependent variables were standardized ( $\beta_{\mathrm{DV}}$ 's) are given in parenthesis. The degrees of freedom, $R^{2}$ changes, and $R^{2}$ of the full model are also given. We see that students whose reasons for studying physics became more autonomous over the duration of the course (i.e., more because they wanted to versus had to) tended to perform better in the course $(N=162)$.

\begin{tabular}{llc}
\hline \hline & & Course grade \\
\hline Step 1 & GPA & $0.57^{* * *}$ \\
& Female & $-0.24^{* * *}(-0.49)$ \\
Step 2 & Instructor 1 & $-0.070(-0.23)$ \\
& Instructor 2 & $-0.13(-0.32)$ \\
& Instructor 3 & $-0.19^{*}(-0.47)$ \\
& Instructor 4 & $-0.29^{* * *}(-0.66)$ \\
Step 3 & RAI Change Score & $0.17^{*}$ \\
& Degrees of freedom & $(7,154)$ \\
& $\left(R^{2}\right)$ change for step 2 & $0.05^{*}$ \\
& $\left(R^{2}\right)$ change for step 3 & $0.03^{* *}$ \\
& $\left(R^{2}\right)$ of full model & $0.41^{* * *}$ \\
\hline \hline
\end{tabular}


$[\beta(8,153)=0.15,95 \% \mathrm{CI}[0.01,0.30]]$ and the change in $R^{2}$ upon adding RAI Change Score to the model in the last step remained statistically significant (change in $\left.R^{2}=0.02, p=0.025\right)$. Thus, Course Grade was correlated with RAI Change Score even after controlling for the effects of IAS.

Finally, we reran the model including the instructor variables in the last step and again found a statistically significant change in $R^{2}$ upon including the instructor variables [whether we controlled for IAS (change in $R^{2}=0.05, p=0.022$ ) or not (change in $R^{2}=0.06$, $p=0.007)$ ], suggesting that differences among instructors had a statistically significant effect on Course Grade that was not attributable to RAI Change Score or IAS.

\section{DISCUSSION}

In this section, we discuss the main findings of this study. We first consider each of our four hypotheses in turn and then discuss the effects of differences among instructors. We also compare our findings to those of the introductory organic chemistry course of BD00 throughout.

\section{A. Discussion of hypothesis 1}

Hypothesis 1 was substantiated by our finding that students who initially took the course for relatively more autonomous reasons (e.g., to learn and understand the material) had higher interest/enjoyment in learning physics (a medium size effect) and were more focused on learning (versus grades) at the end of the course (a small to medium size effect), suggesting a more positive experience throughout the course.

Other work in PER has demonstrated the benefits of a learning (versus performance) orientation with a different population. Reference [52] found that practicing physicists and chemists who reported a learning orientation as their reason for going to graduate school were more productive (as measured by primary and/or first-author publications and grant funding) than those reporting a performance orientation. The correlations mentioned here provide further support for efforts by educators and educational institutions to encourage and facilitate autonomous reasons for taking physics courses and engaging in course activities.

Interest/Enjoyment and Grade Orientation are perhaps the least surprising of the four adjustment variables to be correlated with initial relative autonomous reasons for studying physics, as students taking the course for relatively more autonomous reasons (i.e., more because they want to, less because they have to) likely find the material more interesting/enjoyable and are thus less likely to be grade (versus learning) oriented. The two correlations are in agreement with the findings of BD00; however, BD00 also found that relatively more autonomous reasons for studying predicted significantly higher perceived competence and lower anxiety. Thus, it seems that initial relative autonomous reasons for studying were more influential with regards to anxiety and perceived competence for the BD00 population.

\section{B. Discussion of hypothesis 2}

Hypothesis 2 was substantiated by our finding that perceived instructor autonomy support was positively correlated with increases over the duration of the quarter in interest/enjoyment in learning physics (a medium size effect), decreases in anxiety about taking physics (a small to medium size effect), and increases in relative autonomous reasons for studying physics (a small to medium size effect).

As correlation is not causation, we cannot distinguish the interpretation that higher perceived instructor autonomy support led to these more favorable changes as posited by SDT, from the interpretation that students who had more favorable changes tended to perceive their instructors as more autonomy supportive (perhaps, e.g., instructors responded to increased interest with more autonomy support or they tried to help students who appeared more anxious by giving more direction and less autonomy support). If the former were true, then efforts to enhance instructor autonomy support would be rewarded with more favorable changes in student interest, anxiety, and relative autonomous reasons for studying physics. If the latter were true, then efforts to increase student interest and relative autonomous reasons and decrease anxiety would be rewarded with more autonomy-supportive perceptions of the instructor. Both the former and the latter are positive situations, and both require that the course allows for a significant number of potentially autonomy-supportive instructor-student interactions.

The interpretation that increased instructor autonomy support caused a more favorable student experience in the course is consistent with experimental studies such as that of Ref. [39], which found that increased autonomy support in 20 high school teachers as a result of a training intervention caused increased engagement in their students, where engagement included observed interest and enjoyment in a task as well as attention, effort, participation, and persistence. This causal interpretation would suggest that, in settings similar to the present study, efforts by instructors to adopt specific autonomy-supportive behaviors can significantly improve their students' affective experiences in physics. Greater interest/enjoyment in physics and decreased anxiety about taking physics could result in various desirable consequences, including increased enrollment in physics. The numerous positive associations with more autonomous reasons for learning in educational settings were discussed in Sec. II B.

The above-mentioned correlations are consistent with those of BD00. We do note, however, a large difference between the mean leader autonomy support (LAS) score of $\mathrm{BD} 00$ at $\mathrm{T} 2$ and the mean IAS score of this study. LAS of BD00 is equivalent to IAS of the present study with the exception that IAS included one less item from the LCQ 
(as discussed in Sec. IV B and study II). Given this difference, we compared LAS and IAS as percentages of their maximum possible score, yielding $59.6 \pm 0.9 \%$ $(\mathrm{M} \pm \mathrm{SEM})$ for LAS (T2) and $82.2 \pm 0.9 \%$ for IAS. This very large difference (Cohen's $d=1.98,95 \%$ CI $[1.71,2.25])$ suggests that the instructors in the introductory physics course of the present study were perceived by students as much more autonomy supportive than the instructors of the introductory organic chemistry course of BD00. Despite this large difference, IAS in this study exhibited similar correlations with the dependent variables as LAS did in BD00, with one exception for performance that we discuss next.

\section{Discussion of hypothesis 3}

Hypothesis 3 was substantiated by our finding that instructor autonomy support as perceived by the students was correlated with better performance only for students initially studying physics for relatively autonomous reasons (a medium size effect). There was no correlation for those relatively low in autonomous reasons for studying physics.

Again, we cannot determine the direction of causation in the correlation for those relatively high in autonomous reasons for studying physics, i.e., whether perceiving one's instructor as more autonomy supportive resulted in higher performance or higher performance resulted in a more autonomy-supportive perception of one's instructor (e.g., perhaps instructors provided more autonomy support to high performers). BD00, however, did highlight two findings that argue against the interpretation that higher performance caused higher IAS. First, BD00 found, as we did, that more favorable changes in adjustment were correlated with higher perceived autonomy support (hypothesis 2) even after controlling for performance. This means that the correlation of adjustment changes with IAS was not simply caused by the fact that adjustment changes and IAS were each individually correlated with performance. In other words, there was a significant amount of covariance in the student perception variables (IAS and adjustment) that was not caused by student performance. Second, the average performance of the students of each of the group leaders in BD00 was correlated with each group leader's average perceived autonomy support. Given that students were randomly assigned to each group leader, these differences in performance must be a result of the group leaders. The fact that instructors caused differences in performance that were correlated with perceived autonomy support is consistent with perceived autonomy-support-causing performance [61]. These findings provide support for the interpretation that higher IAS caused higher performance (for students initially more autonomously motivated). This causal interpretation is consistent with experimental studies [39], and would provide support for autonomy-supportive instructor behaviors (see Sec. IIC) in college-level introductory physics courses like that of the present study.

BD00 was the first to find that students' initial relative autonomous reasons for taking the course moderated the correlation of perceived instructor autonomy support with performance; however, in BD00 the correlation was significant for students relatively low in autonomous reasons for studying physics and not for those relatively high, the opposite of the present study. Let us assume that higher perceived autonomy support caused higher performance and consider some differences between BD00 and the present study that may have played a role in the different findings. A major difference between the introductory organic chemistry workshops of BD00 and the introductory physics DLs of this study is that the workshops were smaller and more intimate environments, 6-8 students per peer leader versus approximately 30 students per DL instructor. Students in workshops likely had more one-on-one interactions with their peer leaders than students in DLs had with their instructors. Also, according to Ref. [62], peer leaders in the workshop chemistry project often assume less of a traditional authoritative instructor roll and instead more of a mentor or role model role. In addition, some peer leaders were undergraduates and probably felt like they were more on the same level with their students than DL instructors who were all graduate students.

BD00 suggested that "Providing interpersonal support for students who were not volitional in taking this natural science course seems to have been quite important for them to do well in the course, although students who were autonomously motivated did not seem to need the instructor support in order to do well." A possible explanation of the above-mentioned difference between the findings of BD00 and the present study is as follows. Perhaps "acknowledge perspectives and feelings" is the more salient component of instructor autonomy support in a more intimate environment like the workshops and has a larger effect on the performance of those relatively low in autonomous reasons for taking the course. For example, one might expect acknowledging the feelings of students who are studying physics to avoid negative external consequences or because of internal pressures (controlled reasons) to have a larger effect on performance than for students more autonomously motivated. Perhaps "provide opportunities for choice while minimizing external pressures" is the more salient component of instructor autonomy support in a somewhat less intimate environment like the DLs and has a larger effect on the performance of those relatively high in autonomous reasons for taking physics. For example, one might expect students taking physics because of a desire to learn the material (an autonomous reason) to be more likely to take advantage of opportunities for choice in their interactions with their instructor to guide their experience toward increased learning (e.g., by asking questions, guiding class discussions, etc.). Whereas for students taking the course for 
more controlled reasons (e.g., others' perceptions of them), one might expect the opportunities for choice in their interactions with their instructor to be less likely to affect how well they learn the material.

\section{Discussion of hypothesis 4}

Hypothesis 4 was substantiated by our finding that more positive changes over the duration of the quarter in relative autonomous reasons for studying physics were correlated with better performance (a small to medium size effect), in agreement with BD00.

We took this a step further and investigated whether this correlation was entirely due to the correlation of perceived instructor autonomy support with both more positive changes in relative autonomous reasons for studying physics and performance (as discussed above). We found that it could not be explained entirely by correlations with instructor autonomy support. Thus, those students whose reasons for studying physics became more autonomous tended to perform better, independent of the level of perceived instructor autonomy support.

To summarize the correlations with performance in our findings, for students initially relatively low in autonomous reasons for studying physics, perceived instructor autonomy support was associated with better performance; for all students combined, perceived instructor autonomy support was associated with students' reasons for studying physics becoming relatively more autonomous over the quarter, which in turn was associated with better performance. Assuming the direction of causation implied by self-determination theory, these findings argue for the adoption of autonomy supportive behaviors by physics instructors in order to enhance student performance in courses and with students like those of the present study.

\section{E. Differences among instructors}

Here, we summarize and discuss the effects of differences among instructors. We found a statistically significant difference in perceived instructor autonomy support among the five instructors included in this study, suggesting that student-perceived instructor autonomy support is to some extent measuring an instructor effect (in agreement with, e.g., Ref. [43]).

Additionally, differences among instructors accounted for a statistically significant amount of the variance in changes in students' relative autonomous reasons for studying physics, above and beyond the correlation with perceived instructor autonomy support. So not only was perceived autonomy support associated with relatively more autonomous reasons for studying physics, but other aspects of instructors also had a significant effect on the degree to which students' reasons for studying physics became autonomous. This is clearly important given the many associations with more autonomous reasons in students (improved performance in this study; see Sec. II B for other studies).
For students entering the course relatively low in autonomous reasons for studying physics, differences among instructors accounted for a statistically significant amount of the variance in student performance, above and beyond the correlation with perceived instructor autonomy support. This could have been due to a variety of instructor differences (familiarity with the content, amount of teaching experience, etc.) that for some reason affected those relatively low in autonomous reasons more. This finding that instructors had an effect on the performance of those initially relatively low in autonomous reasons for studying physics may be related to the BD00 finding that performance was correlated with perceived instructor autonomy support only for those initially relatively low in autonomous reasons for studying chemistry.

The above differences among instructors may have been due to classroom influences other than those of the instructor (i.e., classroom culture, time of day, etc.). Given that each instructor except instructor 1 taught two sections, the mean value of each instructor is an average over two classrooms, thus increasing the likelihood that differences among instructors were due to instructor influences. This interpretation is consistent with the findings of Ref. [63], which investigated instructor differences in Physics 7A, 7B, and 7C by observing and documenting the behaviors of 29 DL instructors during Winter quarter 20072008. She found a great deal of variation among instructors in types of behaviors. She also found that the fraction of time instructors spent actively observing (i.e., listening to) students, as students worked in groups, was positively correlated with the final exam performance of their students. This sort of effect could be partially responsible for some of the above-mentioned instructor differences.

\section{CONCLUSION}

In this section we summarize our main findings, discuss the relevance of our findings to other research, consider the limitations of this study and opportunities for future work, and discuss possible implications of our findings.

\section{A. Summary of main findings}

We investigated how various aspects of the student experience in introductory physics were correlated with how autonomy supportive students perceived their instructors to be. We did so to better understand what instructors can do in their interactions with students to enhance student autonomy and thus improve the student experience. Our main findings confirm our four hypotheses (see Sec. III) as follows.

Hypothesis 1 was confirmed by our finding that entering the course with more autonomous (versus controlled) reasons for studying physics predicted a better student experience as measured by higher interest/enjoyment in learning the material and less of a focus on grades (versus learning) during the course. 
Hypotheses 2 was confirmed by our finding that students who perceived their instructors as more autonomy supportive tended to become more autonomous in their reasons for studying physics (which in turn correlated with performance, hypothesis 4), become less anxious about taking physics, and increase in their interest/enjoyment in learning physics.

Hypothesis 3 was confirmed by our finding that students who perceived their instructors as more autonomy supportive tended to perform better in the course, depending on their initial level of relative autonomous reasons for participating in the course.

Hypothesis 4 was confirmed by our finding that students whose reasons for studying physics became relatively more autonomous tended to perform better in the course.

In summary, more autonomous forms of motivation were associated with a better student experience and improved academic outcomes (hypotheses 1 and 4), and students who perceived their instructors as more autonomy supportive tended to have a more favorable experience in the course (hypotheses 2 and 3). These findings are consistent with self-determination theory and previous work in other educational settings (see Sec. II).

\section{B. Relevance to other work}

Previous work in the field of PER has identified student autonomy (which they described as students having input in instructional decisions) as an instructional practice not widely embraced by the PER community that merits serious investigation by the community [46]. As one of a few studies to investigate the SDT concept of autonomy support in a college-level natural science course (BD00 in organic chemistry being the first) and the first to our knowledge in a collegelevel physics course, this study is a step in that direction.

In addition to shedding light on how perceived instructor autonomy support was associated with the student experience, the present correlational study (study I) also sets the stage for a companion experimental study (study II) [9]. In study II we performed a controlled experiment (with a subset of the students from study I) investigating how restructuring the course, to allow more choices by students, affected the student experience. Thus, study II investigates how the course format can be changed to support student autonomy and improve the student experience. Study I, on the other hand, investigates how instructors, in their interactions with students, can better support student autonomy and improve the student experience. The results of study I are used as controls in study II to isolate the effects, on the student experience, of the amount of student choice designed into the course format [9].

The present study builds on the previous work of BD00 in organic chemistry by providing important information regarding the external validity of their findings and the relevance of autonomy support to introductory physics courses like that of the present study. Those findings listed in Sec. VII A are shared with BD00, and have much higher external validity given the different universities, student populations, fields of study, course formats, numbers of instructors, sets of researchers, etc. of the two studies.

The following findings were not shared between BD00 and this work and are thus more context dependent. Entering the course with more autonomous reasons for studying physics predicted higher perceived competence and lower anxiety during the course in BD00 but not in the present study. Additionally, perceived instructor autonomy support was positively correlated with higher performance only for those students whose autonomous reasons for taking the course were relatively high in this work and only for those relatively low in BD00.

\section{Limitations and future work}

An important limitation of the correlational research design of this work is that causation cannot be determined from our perceived instructor autonomy support correlations. To determine if instructor autonomy support in fact causes a more favorable student experience would require an experimental research design comparing dependent variables (adjustment, performance, etc.) across experimental and control groups with different levels of instructor autonomy support, while controlling for all other variables that may affect the dependent variables. To isolate the cause of any measured differences in the dependent variables would require information about how, specifically, instructor behaviors in the experimental and control groups were different. This information could be obtained by observing and comparing instructor behaviors (e.g., by observing validated autonomy supportive behaviors $[30,64]$ or using a climate questionnaire as a rating device [65]). It would not be sufficient to compare how autonomy supportive students perceived their instructors to be, though this could provide information about how student perceptions mediated any observed effects (e.g., as in Ref. [30]).

Experimental studies have found that increased autonomy support in fact caused such positive effects as increased engagement in the students of high school teachers [39], more perceived competence and autonomous motivation for dentalhealth care and improved dental health, self-care behavior, attitudes, and affect in the patients of dental hygienists [66], and more autonomous motivation and workplace engagement in managers' employees [67]. However, to our knowledge, no experimental studies investigating the effects of instructor autonomy support on the student experience have been carried out in a college-level natural science course like that of the present study. This is an avenue for future research.

A second possible limitation of this correlational study relates to the reliability of our measures as commonly estimated by Cronbach's alpha, a coefficient of internal consistency. Alpha is a function of both the number of items composing a measure and the sample size (see, e.g., Ref. [68]). Reference [68] provided a matrix for estimating the adequacy of alpha values, based on the number of items 
and sample size. The matrix serves as a general guide, where values at or above suggested cutoffs may be considered adequate. Following these guidelines, most administrations of measures in this study had alphas above the cutoffs (Interest/Enjoyment, Perceived Competence, Anxiety, and Instructor Autonomy Support were well above the cutoffs). The following administrations of three of our measures did not. The suggested cutoff for the Grade Orientation Measure was 0.65 . Alpha was 0.63 at $\mathrm{T} 1$ (the $\mathrm{T} 2$ administration was above the cutoff). The suggested cutoff for the General Causality Orientations Subscales was 0.75. Alphas were $0.74,0.66$, and 0.72 for the autonomous, controlled, and impersonal subscales, respectively. We note that these subscales were used as control variables only in this study. Finally, the suggested cutoff for the controlled subscale of the learning Self-Regulation Questionnaire (one component of the Relative Autonomy Index) was 0.70. Alpha was 0.60 at T1 (alpha was above the cutoff at T2). Measures with low reliability (as a result of high error variance) can underestimate the effect sizes of relationships between the constructs the measures operationalize (see, e.g., Refs. [68,69]). Thus, a possible limitation of this study is that effect sizes were underestimated for correlations between constructs these three measures investigated.

\section{Implications}

Higher instructor autonomy support as perceived by the students was correlated with an overall better experience in the course for the introductory physics students of this study (and the introductory organic chemistry students of BD00). If higher instructor autonomy support in fact caused a better student experience, as suggested by self-determination theory and experimental studies in other contexts, these results would have implications for instructors, instructor professional developers, and course designers in similar introductory college science courses.
These results would argue for the combination of (1) course designs that provide students with many opportunities for autonomy-supportive interactions with their instructors (e.g., not traditional large lecture-based courses; BD00) and (2) instructors that are autonomy supportive in their interactions with students. Simply reformatting a course to allow more instructor-student interactions would not be enough; instructors would need to take advantage of the increased number of interactions with students to support their autonomy.

These results would also argue for incorporating the concept of autonomy support into instructor professional development efforts. The autonomy-supportive instructor interactions investigated in this study were acknowledging students' perspectives and feelings and providing students with relevant information and opportunities for choice while minimizing external influences and pressures $[22,55]$. For information (primarily in K-12 contexts) about other aspects of autonomy support, how instructors can implement autonomy support in the classroom, and professional development efforts to help people support the autonomy of others, see, for example, the work of Reeve and co-workers ([23,24,29,70], and references therein).

Increased interest/enjoyment in learning physics, more autonomous reasons for studying physics (i.e., more because students want to versus have to), decreased anxiety about taking physics, and better performance would certainly make the above-mentioned efforts by educators worthwhile.

\section{ACKNOWLEDGMENTS}

We would like to thank the UC Davis Physics Education Research Group, particularly Robert Lynch, Cassandra Paul, Wendell Potter, and Antoinette Stone, for their helpful insights and feedback. We would also like to thank the instructors and students who took part in this study for generously giving of their time.
[1] E. L. Deci and R. M. Ryan, Intrinsic Motivation and SelfDetermination in Human Behavior (Plenum, New York, 1985).

[2] E. L. Deci and R.M. Ryan, The "what" and "why" of goal pursuits: Human needs and the self-determination of behavior, Psychological inquiry 11, 227 (2000).

[3] R. M. Ryan and E. L. Deci, Self-determination theory and the facilitation of intrinsic motivation, social development, and well-being, Am. Psychol. 55, 68 (2000).

[4] W. Potter, D. Webb, C. Paul, E. West, M. Bowen, B. Weiss, L. Coleman, and C. D. Leone, Sixteen years of collaborative learning through active sense-making in physics (CLASP) at UC davis, Am. J. Phys. 82, 153 (2014).
[5] P. W. Laws, CalculusBased physics without lectures, Phys. Today 44, No. 12, 24 (1991).

[6] P. W. Laws, Workshop Physics Activity Guide (John Wiley \& Sons, New York, 1997).

[7] National Science Board, National Science Foundation, Technical Report No. NSB 12-01, 2012.

[8] A. V. Maltese and R. H. Tai, Pipeline persistence: Examining the association of educational experiences with earned degrees in STEM among U.S. students, Sci. Educ. 95, 877 (2011).

[9] N. R. Hall, "Autonomy and the student experience in introductory physics," Ph.D. thesis, University of California, Davis, 2013 (http://pqdtopen.proquest.com/ pubnum/3602079.html). 
[10] R. M. Ryan and E. L. Deci, Intrinsic and extrinsic motivations: Classic definitions and new directions, Contemp. Educ. Psychol. 25, 54 (2000).

[11] P. J. Silvia, "Interest-The curious emotion," Curr. Dir. Psychol. Sci. 17, 57 (2008).

[12] G. Loewenstein, The psychology of curiosity: A review and reinterpretation, Psychol. Bull. 116, 75 (1994).

[13] R. M. Ryan and J. P. Connell, Perceived locus of causality and internalization: Examining reasons for acting in two domains, J. Pers. Soc. Psychol. 57, 749 (1989).

[14] J. G. Nicholls, Achievement motivation: Conceptions of ability, subjective experience, task choice, and performance, Psychol. Rev. 91, 328 (1984).

[15] R. M. Ryan, Control and information in the intrapersonal sphere: An extension of cognitive evaluation theory, J. Pers. Soc. Psychol. 43, 450 (1982).

[16] C. P. Niemiec and R. M. Ryan, Autonomy, competence, and relatedness in the classroom: Applying selfdetermination theory to educational practice, Theory Res. Educ. 7, 133 (2009).

[17] M. Miserandino, Children who do well in school: Individual differences in perceived competence and autonomy in above-average children, J. Educ. Psychol. 88, 203 (1996).

[18] W. S. Grolnick, R. M. Ryan, and E. L. Deci, Inner resources for school achievement: Motivational mediators of children's perceptions of their parents, J. Educ. Psychol. 83, 508 (1991).

[19] M. Standage, J. L. Duda, and N. Ntoumanis, Students' motivational processes and their relationship to teacher ratings in school physical education, Res. Q. Exercise Sport 77, 100 (2006).

[20] C. P. Niemiec, M. F. Lynch, M. Vansteenkiste, J. Bernstein, E. L. Deci, and R. M. Ryan, The antecedents and consequences of autonomous self-regulation for college: A selfdetermination theory perspective on socialization, J. Early Adolesc. 29, 761 (2006).

[21] A.E. Black and E. L. Deci, The effects of instructors' autonomy support and students' autonomous motivation on learning organic chemistry: A self-determination theory perspective, Sci. Educ. 84, 740 (2000).

[22] G. C. Williams and E. L. Deci, Internalization of biopsychosocial values by medical students: A test of selfdetermination theory, J. Pers. Soc. Psychol. 70, 767 (1996).

[23] Y.-L. Su and J. Reeve, A meta-analysis of the effectiveness of intervention programs designed to support autonomy, Educ. Psychol. Rev. 23, 159 (2011).

[24] J. Reeve, Why teachers adopt a controlling motivating style toward students and how they can become more autonomy supportive, Educ. Psychol. 44, 159 (2009).

[25] J. Reeve, H. Jang, P. Hardre, and M. Omura, Providing a rationale in an autonomy-supportive way as a strategy to motivate others during an uninteresting activity, Motivation and emotion 26, 183 (2002).

[26] M. Vansteenkiste, J. Simons, W. Lens, K. M. Sheldon, and E. L. Deci, Motivating learning, performance, and persistence: The synergistic effects of intrinsic goal contents and autonomy-supportive contexts, J. Pers. Soc. Psychol. 87, 246 (2004).
[27] M. Vansteenkiste, J. Simons, W. Lens, B. Soenens, and L. Matos, Examining the motivational impact of intrinsic versus extrinsic goal framing and autonomy-supportive versus internally controlling communication style on early adolescents' academic achievement, Child Development 76, 483 (2005).

[28] A. Assor, H. Kaplan, Y. Kanat-Maymon, and G. Roth, Directly controlling teacher behaviors as predictors of poor motivation and engagement in girls and boys: The role of anger and anxiety, Learn. Instr. 15, 397 (2005).

[29] J. Reeve and H. Jang, What teachers say and do to support students' autonomy during a learning activity, J. Educ. Psychol. 98, 209 (2006).

[30] J. Reeve, G. Nix, and D. Hamm, Testing models of the experience of self-determination in intrinsic motivation and the conundrum of choice, J. Educ. Psychol. 95, 375 (2003).

[31] E. A. Patall, H. Cooper, and J. C. Robinson, The effects of choice on intrinsic motivation and related outcomes: A meta-analysis of research findings, Psychol. Bull. 134, 270 (2008).

[32] S. Williams, An organizational model of choice: A theoretical analysis differentiating choice, personal control, and self-determination, Genetic, social, and general psychology monographs 124, 465 (1998).

[33] T. Flowerday, G. Schraw, and J. Stevens, The role of choice and interest in reader engagement, J. Exp. Educ. 72, 93 (2004).

[34] I. Katz and A. Assor, When choice motivates and when it does not, Educ. Psychol. Rev. 19, 429 (2007).

[35] C. R. Stefanou, K. C. Perencevich, M. DiCintio, and J.C. Turner, Supporting autonomy in the classroom: Ways teachers encourage student decision making and ownership, Educ. Psychol. 39, 97 (2004).

[36] E. L. Deci, Effects of externally mediated rewards on intrinsic motivation, J. Pers. Soc. Psychol. 18, 105 (1971).

[37] M. R. Lepper, D. Greene, and R. E. Nisbett, Undermining children's intrinsic interest with extrinsic reward: A test of the "overjustification" hypothesis, J. Pers. Soc. Psychol. 28, 129 (1973).

[38] E. L. Deci, R. Koestner, and R. M. Ryan, A meta-analytic review of experiments examining the effects of extrinsic rewards on intrinsic motivation, Psychol Bull. 125, 627 (1999).

[39] J. Reeve, H. Jang, D. Carrell, S. Jeon, and J. Barch, Enhancing students' engagement by increasing teachers' autonomy support, Motivation and emotion 28, 147 (2004).

[40] A. Assor, G. Roth, and E. L. Deci, The emotional costs of parents' conditional regard: A self-determination theory analysis, J. Pers. 72, 47 (2004).

[41] J. Reeve, Self-determination theory applied to educational settings, in The Handbook of Self-Determination Research, 1st ed., edited by E. L. Deci and R. M. Ryan (University of Rochester Press, Rochester, NY, 2002), pp. 183-203.

[42] E. L. Deci, A. J. Schwartz, L. Sheinman, and R. M. Ryan, An instrument to assess adults' orientations toward control versus autonomy with children: Reflections on intrinsic motivation and perceived competence, J. Educ. Psychol. 73, 642 (1981). 
[43] R. M. Ryan and W. S. Grolnick, Origins and pawns in the classroom: Self-report and projective assessments of individual differences in children's perceptions, J. Pers. Soc. Psychol. 50, 550 (1986).

[44] Y.-M. Tsai, M. Kunter, O. Ldtke, U. Trautwein, and R. M. Ryan, What makes lessons interesting? The role of situational and individual factors in three school subjects, J. Educ. Psychol. 100, 460 (2008).

[45] The effect size of each study was weighted by the study's sample size.

[46] M. Dancy and C. Henderson, Framework for articulating instructional practices and conceptions, Phys. Rev. ST Phys. Educ. Res. 3, 010103 (2007).

[47] D. Gosser, V. Roth, L. Gafney, J. Kampmeier, V. Strozak, P. Varma-Nelson, S. Radel, and M. Weiner, Workshop chemistry: Overcoming the barriers to student success, Chem. Educ. 1, 1 (1996).

[48] By controlling for instructor differences, we investigated correlations of within-instructor differences in the dependent variables (e.g., performance) with withininstructor differences in the independent variables (e.g., instructor autonomy support). BD00 did not control for instructors' differences and thus investigated the combination of within-instructor and between-instructor differences.

[49] Particularly, instructor 5 would begin DL with discussions unrelated to course content and wait for students to initiate course-related activities. This continued throughout the DL, with instructor 5 not proactively guiding students.

[50] C. D. Spielberger, P. R. Vagg, L. R. Barker, G. W. Donham, and L. G. Westberry, The factor structure of the state-trait anxiety inventory, in Stress and Anxiety (Hemisphere, Washington, DC, 1980), pp. 244-279.

[51] As this was the only four-item measure included in the questionnaire (all others were five), $15 \%$ and $19 \%$ of the students at $\mathrm{T} 1$ and $\mathrm{T} 2$, respectively, responded on a 5-point rather than a 4-point scale on at least one of the items. We rescaled these students' scores to a 4-point scale. Doing so resulted in no noticeable change in our results.

[52] Z. Hazari, G. Potvin, R. H. Tai, and J. Almarode, For the love of learning science: Connecting learning orientation and career productivity in physics and chemistry, Phys. Rev. ST Phys. Educ. Res. 6, 010107 (2010).

[53] See Supplemental Material at http://link.aps.org/ supplemental/10.1103/PhysRevSTPER.10.020116 for the adjustment measures used in this work.

[54] E. L. Deci and R. M. Ryan, The general causality orientations scale: Self-determination in personality, Journal of research in personality 19, 109 (1985).
[55] G. C. Williams, V. M. Grow, Z. R. Freedman, R. M. Ryan, and E. L. Deci, Motivational predictors of weight loss and weight-loss maintenance, J. Pers. Soc. Psychol. 70, 115 (1996).

[56] http://selfdeterminationtheory.org/questionnaires.

[57] F.E. Satterthwaite, An approximate distribution of estimates of variance components, Biometrics Bull. 2, 110 (1946).

[58] J. Cohen, A power primer, Psychol Bull. 112, 155 (1992).

[59] This is desirable as it accounts for differences in changes in adjustment among instructors, which may or may not be due to differences in instructor autonomy support.

[60] Investigated by comparing the error of the slope of the bestfit lines in Fig. 2 to the error of the IAS correlation coefficients in the actual model.

[61] One could imagine, however, a third instructor variable that resulted in differences in both performance and perceived autonomy support.

[62] D. K. Gosser and V. Roth, The workshop chemistry project: Peer-led team-learning, J. Chem. Educ. 75, 185 (1998).

[63] C. A. Paul, Investigation of the interactions between instructors and students in an introductory interactiveengagement college physics course, Ph.D. thesis, University of California, Davis, 2013.

[64] J. Reeve, E. Bolt, and Y. Cai, Autonomy-supportive teachers: How they teach and motivate students, J. Educ. Psychol. 91, 537 (1999).

[65] G. C. Williams and E. L. Deci, Activating patients for smoking cessation through physician autonomy support, Medical care 39, 813 (2001).

[66] A. E. M. Halvari and H. Halvari, Motivational predictors of change in oral health: An experimental test of selfdetermination theory, Motivation and emotion 30, 294 (2006).

[67] P. L. Hardr and J. Reeve, Training corporate managers to adopt a more autonomy-supportive motivating style toward employees: An intervention study, Int. J. Train. Dev. 13, 165 (2009).

[68] J. G. Ponterotto and D. E. Ruckdeschel, An overview of coefficient alpha and a reliability matrix for estimating adequacy of internal consistency coefficients with psychological research measures, Percept. Mot. Skills 105, 997 (2007).

[69] F. Baugh, Correcting effect sizes for score reliability: A reminder that measurement and substantive issues are linked inextricably, Educ. Psychol. Meas. 62, 254 (2002).

[70] J. Reeve, Teachers as facilitators: What autonomysupportive teachers do and why their students benefit, Elem. School J. 106, 225 (2006). 